\title{
Relative-Intensity-Noise Coupling in Heterodyne Interferometers
}

\author{
Lennart Wisselø, ${ }^{1,}{ }^{*}$ Andreas Wittchen $\odot,{ }^{1}$ Thomas S. Schwarze $\odot,{ }^{1}$ Martin Hewitson $\odot,{ }^{1}$ \\ Gerhard Heinzel $\odot,{ }^{1}$ and Hubert Halloin $\circledast^{2}$ \\ ${ }^{1}$ Albert-Einstein-Institut, Max-Planck-Institut für Gravitationsphysik und Leibniz Universität Hannover, \\ Callinstraße 38, Hannover 30167, Germany \\ ${ }^{2}$ Université de Paris, CNRS, Astroparticule et Cosmologie, Paris F-75013, France
}

(Received 12 October 2021; revised 20 December 2021; accepted 21 December 2021; published 9 February 2022)

Laser interferometers are the core measurement tool in gravitational wave observatories. An important factor that can limit the performance is the relative power instability of the laser, a problem often called relative intensity noise (RIN). But exactly how this influences the interferometer performance is not completely understood. Therefore in this paper we analyze laser RIN coupling into the phase readout in balanced and unbalanced heterodyne interferometers. We describe the coupling theoretically, then simulate and finally measure it. Our results reveal a combination of RIN contributions from the heterodyne frequency and twice the heterodyne frequency in the interferometric phase readout. We also show that when an additional, correlated reference measurement is subtracted the combined coupling factor depends on the differential phase between the two measurements and thus can be minimized. Our results have implications for noise models in future space-based gravitational wave observatories like Laser Interferometer Space Antenna, where RIN-to-phase coupling arises directly and is modulated via spacecraft jitter, testmass position and orientation.

DOI: 10.1103/PhysRevApplied.17.024025

\section{INTRODUCTION}

Optical interferometers are commonly used in highprecision metrology and they form, for example, the core measurement tool in gravitational wave observatories [1-4] and are also used for inter-satellite ranging [5]. The measurement tracks the phase difference between two interfering beams which represents an optical pathlength change. In a homodyne readout the interfering beams share the same frequency, whereas in heterodyne detection the two beams are shifted in frequency such that they create a time-varying beat signal through interference at the difference frequency. This signal can be processed with phase demodulation techniques to measure a differential length change [6].

The space-based Laser Interferometer Space Antenna (LISA) mission has to use the heterodyne interferometer technique due to the orbital motion of the three spacecraft (causing Doppler shifts), while measuring the effect of gravitational waves passing through the constellation.

\footnotetext{
*lennart.wissel@aei.mpg.de
}

Published by the American Physical Society under the terms of the Creative Commons Attribution 4.0 International license. Further distribution of this work must maintain attribution to the author(s) and the published article's title, journal citation, and DOI. Open access publication funded by the Max Planck Society.
Closely related, the European Spacer Agency's technology demonstrator mission, LISA Pathfinder (LPF), launched in 2015, and the inter-spacecraft laser ranging interferometer aboard the GRACE Follow-On mission, launched in 2018, also used a similar heterodyne technique [7-10].

One of the technical noise sources in these systems arises from fluctuations of the laser power itself, called relative intensity noise (RIN) or relative power noise. It is typically defined as the power fluctuations in relation to the mean power.

Depending on the requirements, it can be a limiting factor in the interferometric sensitivity, since it couples into the observable of interest, the differential phase, as is shown in this paper.

To mitigate the effect of these perturbations the beam power is typically stabilized before propagating the beam through the interferometer and the remaining excess noise is often removed by applying a balanced detection scheme utilizing two interferometer output ports $[9,11]$.

The scheme for such an interferometer is shown in Fig. 1, where the distance variations between two test masses (TMs) are measured, as was done on LPF.

In this paper we report on the well-known "1f-RIN" (the contribution of RIN from near the heterodyne frequency) and derive coupling relations and its common mode rejection properties. We also analyze a less wellknown "2f-RIN" coupling mechanism (the contribution 


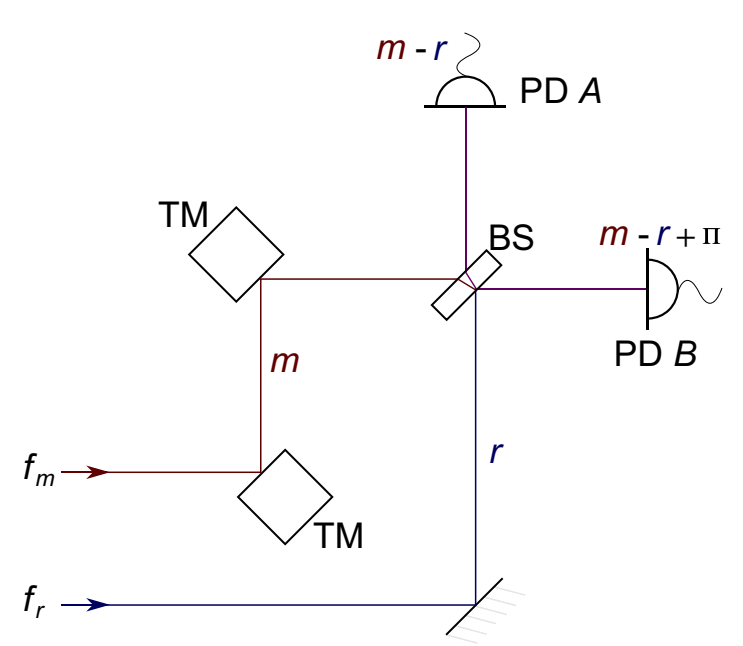

FIG. 1. Simplified heterodyne interferometer. Two beams with a difference frequency $f_{\text {het }}=f_{m}-f_{r}\left(f_{m}>f_{r}\right)$ are brought to interference using a beam combiner [beam splitter (BS)]. The resulting phase signal that corresponds to the arm length difference, $m-r$, is measured in both output ports, A and B, with photodiodes (PDs), which see the signal with a $\pi$ phase shift between them due to energy conservation at the BS. In this case, $f_{m}$ corresponds to the measurement beam with a time-varying phase (the observable of interest being the distance change of the TM), while the propagation path of $f_{r}$ is assumed constant and termed the reference beam. The term "balanced detection" refers to the subtraction of the measurements occurring in PD A and $\mathrm{PD} \mathrm{B}$ to reduce common noise.

of RIN from near twice the heterodyne frequency) to the phase measurement that contributes noise even in a balanced heterodyne interferometer, and the simulations and experiments that were performed to explore these effects in an on-ground laboratory setup.

The "2f-RIN" coupling has partially been described in [11], and similar calculations can be found in [12], but without the exact RIN-to-phase coupling mechanisms.

We extend and build the formulas for both couplings for a number of practical scenarios that are relevant to LISA.

The contribution of the "2f-RIN" effect has also been accounted for in the LPF mission, as described in [7].

Note that radiation pressure noise on the TM due to the effects of low frequency RIN are not detailed in this paper.

\section{OVERVIEW}

To set the scope, this paper begins with a review of the typical heterodyne equations in Sec. III.

The definition of RIN, its modeling and the derivation of the resulting phase noise is described in Sec. IV in great detail.

Computational simulations (Sec. V) and laboratory measurements (Sec. VI) support the theoretical predications.

Especially the theoretical section is complex to read due to all the different scenarios discussed. To aid reading, we anticipate the main RIN-to-phase coupling features in what follows.

The RIN $r$ is defined as fluctuations of the laser power divided by its mean value, that is, $r=\delta P / \bar{P}$. It causes phase noise in optical heterodyne interferometers and there are two (first order) distinct coupling mechanisms: (i) purely additive $1 \mathrm{f}-\mathrm{RIN}$ from around the heterodyne frequency; and (ii) multiplicative (nonlinear) 2f-RIN from around twice the heterodyne frequency that can also be treated as additive noise after Taylor expansion.

a. If-RIN. 1f-RIN describes the coupling of RIN from around the heterodyne frequency. The resulting phase noise depends on beam powers, beam splitter coefficients and the heterodyne efficiency (contrast) and subtracts with (ideal) balanced detection. It scales approximately with $1 /$ contrast and can be magnified by large power mismatches. 1f-RIN common mode rejection shows sinusoidal behavior with respect to the relative phase (e.g., visible via the testmass position). In this case, if the phase difference can be kept close to zero, the coupling is further reduced with respect to the balancing of the beam powers.

b. 2f-RIN. 2f-RIN describes the coupling of RIN from twice the heterodyne frequency. Now, the resulting phase noise is independent of beam powers and contrast, but it does not subtract with balanced detection. It is roughly a factor contrast $/ 2$ smaller than the corresponding $1 \mathrm{f}-\mathrm{RIN}$ term for equal beam powers. $2 \mathrm{f}$-RIN common mode rejection shows sinusoidal behavior with respect to twice the relative phase (e.g., typical noise dependency on testmass position similar $1 \mathrm{f}-\mathrm{RIN})$. In this case, if the phase difference can be kept close to zero, the coupling is minimized (no dependency on the power ratio).

These two distinct coupling mechanisms contribute uncorrelated phase noise (since they are usually far apart in frequency) and thus need to be summed quadratically.

\section{REVIEW OF HETERODYNE INTERFEROMETRY}

Recall that the well-known relations for a heterodyne interferometer with two output ports A, B, can be derived by assuming two Gaussian beams, $\mathbf{E}_{m}, \mathbf{E}_{r}$, propagating through a beam splitter onto two photodiodes, one per output port, as depicted in Fig. 1.

The beams are assumed to be well aligned when hitting the detectors and for simplicity we assume that both beams travel (locally) in the $z$ direction at all times. In fact, the propagation direction of the beams is tilted by $90^{\circ}$ after reflection of the beam splitter, but we neglect any offsets or imperfections in the optical path and perform the calculation for parallel beams.

Our mathematical description for two beams (denoted $m$ for measurement, $r$ for reference beam with indices $k=$ 
$\{m, r\})$ assumes propagation through a vacuum and travel times $t_{k}$ when arriving at the detector:

$$
\begin{aligned}
\mathbf{E}_{m} & =\operatorname{Re}\left\{\mathbf{e}_{p} a_{m}\left(x, y, t-t_{m}\right) e^{i\left[\omega_{m}\left(t-t_{m}\right)\right]}\right\}, \\
\mathbf{E}_{r} & =\operatorname{Re}\left\{\mathbf{e}_{p} a_{r}\left(x, y, t-t_{r}\right) e^{i\left[\omega_{r}\left(t-t_{r}\right)\right]}\right\},
\end{aligned}
$$

with unity polarization vectors $\mathbf{e}_{p}$ and $\mathbf{e}_{p} \perp \mathbf{e}_{z}$, positionand time-dependent amplitudes $a_{m, r}(x, y, t) \in \mathbb{C}$, and optical frequencies $\omega_{k}=2 \pi f_{k}, f_{m}=f_{r}+f_{\text {het }}$. As usual we drop the $\operatorname{Re}\{\cdot\}$ in the following and implicitly assume it for measurable quantities like the electric field.

Typically, $f_{\text {het }}$ is much smaller than $f_{m, r}$, which leads to a slowly varying heterodyne beatnote, while the faster frequencies are averaged away through the detection process. This is implicitly assumed when taking the absolute square (see the following calculations). Constant factors are absorbed in $a_{m, r}$ such that $\left|a_{m, r}\right|^{2}$ represents an intensity with units $\mathrm{Wm}^{-2}$. The detectors are assumed to be located at an equivalent local $z=0$ point in each arm.

We assume a light travel time, $t_{c}$, that is common to both beams, and a slowly time-varying differential time, $t_{d}$, that we attribute to $\mathbf{E}_{m}$ in the sense that the runtime difference of the beams is $t_{m}-t_{r}=\left(t_{c}+t_{d}\right)-t_{c}=t_{d}$, which translates to a slowly time-varying differential phase. This light travel time difference between the beams is magnified by a large optical carrier frequency, as given by $\phi_{d}=\omega_{m} t_{d}$.

We drop the polarization vector, $\mathbf{e}_{p}$, since both beams are assumed to have identical polarization, perpendicular to the plane of incidence of the recombination beam splitter and the detector surface.

The properties of a lossless beam splitter are usually described by amplitude reflection and transmission coefficients, $\rho, \tau \in \mathbb{C}, \rho^{2}+\tau^{2}=1$, which are used in a unitary coupling matrix, $\mathbf{M}$, such that

$$
\mathbf{M}=\left(\begin{array}{cc}
\rho & i \tau \\
i \tau & \rho
\end{array}\right) \quad \text { or } \quad \mathbf{M}=\left(\begin{array}{cc}
\rho & \tau \\
\tau & -\rho
\end{array}\right)
$$

The optical intensities impinging on the photodiodes in ports $A$ and $B$ can then be calculated by squaring the amplitudes $\left(E_{A}, E_{B}\right)^{\top}=\mathbf{M} \cdot\left(E_{m}, E_{r}\right)^{\top}$,

$$
\begin{aligned}
\left|E_{A}\right|^{2} & =\left|\rho E_{m}+\tau E_{r}\right|^{2} \\
& =\rho^{2}\left|E_{m}\right|^{2}+\tau^{2}\left|E_{r}\right|^{2}+2 \rho \tau \operatorname{Re}\left[E_{m} E_{r}^{*}\right], \\
\left|E_{B}\right|^{2} & =\left|\tau E_{m}-\rho E_{r}\right|^{2} \\
& =\tau^{2}\left|E_{m}\right|^{2}+\rho^{2}\left|E_{r}\right|^{2}-2 \rho \tau \operatorname{Re}\left[E_{m} E_{r}^{*}\right],
\end{aligned}
$$

using * to indicate complex conjugation and the fact that $z+z^{*}=2 \operatorname{Re}(z)$.

To describe the detected optical powers, $P_{A, B}$, one has to integrate over the photodiode surface, $S$, such that $P_{A, B}=$ $\int\left|E_{A, B}\right|^{2} d S$.
The first terms of Eq. (3) yield the single-beam powers $\int d S\left(\rho^{2}\left|E_{m}\right|^{2}+\tau^{2}\left|E_{m}\right|^{2}\right)=\int d S\left|E_{m}\right|^{2}=: P_{m}$ for the measurement beam, and $\int d S\left(\tau^{2}\left|E_{r}\right|^{2}+\rho^{2}\left|E_{r}\right|^{2}\right)=$ $\int d S\left|E_{r}\right|^{2}=: P_{r}$ for the reference beam (where $S$ is much greater than the beam diameter).

The integration of the last terms, assuming normalized beam amplitudes $A_{k}(x, y):=a_{k} / \sqrt{P_{k}(t)}$ (again constant factors absorbed in $a_{k}$ ), gives

$$
\begin{aligned}
& \pm 2 \rho \tau \int d S \operatorname{Re}\left[E_{m} E_{r}^{*}\right] \\
& = \pm 2 \rho \tau \sqrt{P_{m} P_{r}} \operatorname{Re}\left[e^{i\left[\omega_{m}\left(t-t_{m}\right)-\omega_{r}\left(t-t_{r}\right)\right]} \int d S A_{m} A_{r}^{*}\right] \\
& = \pm 2 \rho \tau \sqrt{\eta_{\text {het }} P_{m} P_{r}} \cos \left(\omega_{\text {het }} t-\phi_{d}-\phi_{c}-\phi_{\text {het }}\right),
\end{aligned}
$$

with $\omega_{\text {het }}=\omega_{m}-\omega_{r}$ and $\phi_{c}=\omega_{\text {het }} t_{c}$. In the last step, we make use of the so-called overlap integral, $\sigma$, of the two beams (see [13]), arising from beam geometry,

$$
\sigma=\int d S A_{m} A_{r}^{*}=: \sqrt{\eta_{\text {het }}} e^{i \phi_{\text {het }}}
$$

which can be separated into a magnitude factor, $\eta_{\text {het }}=$ $|\sigma|^{2}$, and a phase contribution, $\phi_{\text {het }}=\arg (\sigma)$, to the signal. The amplitude contribution is called the heterodyne efficiency. The additional phase contribution due to the spatial wave front properties is added as a phase factor, which, in general, can be different between the two output ports. We further neglect any time-dependence of the beam geometries.

As a result we arrive at the well-known heterodyne equations with $P_{m, r}=P_{m, r}\left(t-t_{m, r}\right)$ :

$$
\begin{aligned}
& P_{A}=\rho^{2} P_{m}+\tau^{2} P_{r}+2 \rho \tau \sqrt{\eta_{\text {het }, A} P_{m} P_{r}} \times B_{A}, \\
& P_{B}=\tau^{2} P_{m}+\rho^{2} P_{r}-2 \rho \tau \sqrt{\eta_{\text {het }, B} P_{m} P_{r}} \times B_{B},
\end{aligned}
$$

with the beat term, $B_{A, B}$, oscillating at the heterodyne frequency, $f_{\text {het }}$,

$$
\begin{aligned}
B_{A, B} & =\cos \left(\omega_{\text {het }} t-\phi_{d}-\phi_{c}-\phi_{\text {het }, A, B}\right) \\
& =\cos \left(\omega_{\text {het }} t-\varphi_{A, B}\right) .
\end{aligned}
$$

In the last step we merge the geometric phase, $\phi_{\text {het }}$, with $\phi_{d}$ and the typically constant $\phi_{c}$ phase into an effective detected phase signal $\varphi_{A, B}=\phi_{c}+\phi_{d}+\phi_{\text {het }, A, B}$.

To simplify the following sections, we assume no differential losses in the detection process on the photodiodes and neglect differences in the overlap integral between the two ports, so that $\phi_{\text {het }, A, B}=\phi_{\text {het }}, \eta_{\text {het }, A, B}=\eta_{\text {het }}, \varphi=$ $\varphi_{A, B}, B_{A, B}=B$. 
Note that the interferometer contrast can be computed as

$$
\begin{aligned}
& \text { Contrast }_{A}=C_{A}=\frac{2 \rho \tau \sqrt{\eta_{\text {het }, A} P_{m} P_{r}}}{\rho^{2} P_{m}+\tau^{2} P_{r}} \\
& \text { Contrast }_{B}=C_{B}=\frac{2 \rho \tau \sqrt{\eta_{\text {het }, B} P_{m} P_{r}}}{\tau^{2} P_{m}+\rho^{2} P_{r}} .
\end{aligned}
$$

Equations (6) contain two dc terms each, depending on the transmitted and reflected powers, and one signal term per output port, the latter with different signs. This allows us to perform the so-called balanced detection. It is realized by forming the difference between the powers of the two complementary output ports, $\Delta P:=\left(P_{A}-\right.$ $\left.P_{B}\right) / 2$, which reduces excess noise (e.g., certain components of RIN). Ideally for this purpose, $\rho^{2}=\tau^{2}=1 / 2$ with a perfect 50:50 beam splitter.

After interfering and detecting the beams, there are different ways to extract the phase information, for example using phase-locked loops [14] or single-bin discrete Fourier transforms (SBDFTs) [9]. As a practical example, on board LPF the signal was extracted by a Fourier analysis of the form

$$
\begin{gathered}
F=\frac{1}{\pi} \int_{0}^{2 \pi} \Delta P e^{i 2 \pi f_{\text {het }} t} d\left(2 \pi f_{\text {het }} t\right), \\
\Rightarrow=\arg (F)=\arctan \left(\frac{\Im(F)}{\operatorname{Re}(F)}\right) .
\end{gathered}
$$

However, the last equation is only exactly true in the absence of phase noise. How this changes when RIN is introduced into the system will be discussed next.

\section{RIN COUPLING}

RIN (called $r$ ) represents a multiplicative noise source and is usually measured as fluctuations around the mean power:

$$
\begin{aligned}
r(t) & =\delta P / \bar{P}, \\
& \Rightarrow \quad \delta P=r \times \bar{P} \\
& \Rightarrow \quad \bar{P}+\delta P=\bar{P}(1+r),
\end{aligned}
$$

where the bar denotes the mean or average power of the beam. RIN is usually expressed as a spectral density in units $1 / \sqrt{\mathrm{Hz}}$.

Note that one can also derive RIN directly from an amplitude noise, $n$, of the electric field amplitudes in the form $a(t)=\bar{a}(1+n)$, as introduced in Eq. (1). Then $|a(t)|^{2}=\bar{a}^{2}(1+n)^{2} \approx \bar{a}^{2}(1+2 n) \approx \bar{P}(1+r)$, so the above definition matches with RIN $r \approx 2 n$, neglecting the small $n^{2}$ term.

Typical non-planar ring oscillator (NPRO) lasers can be stabilized to operate around or below $1 \times 10^{-4} / \sqrt{\mathrm{Hz}}$ at millihertz and $1 \times 10^{-8} / \sqrt{\mathrm{Hz}}$ at megahertz frequencies, while the heterodyne beat amplitude is assumed to be orders of magnitude larger (see, for example, $[7,15]$ ).

In the following, all powers $P_{k}$ are assumed to be longterm mean values (we refrain from using the extra bar on $\bar{P})$ and only the RIN itself carries the time dependence. Therefore, we introduce RIN denoted by $r_{m, r}(t)$ in the beam powers

$$
P_{m} \rightarrow P_{m}\left[1+r_{m}\left(t-t_{m}\right)\right], \quad P_{r} \rightarrow P_{r}\left[1+r_{r}\left(t-t_{r}\right)\right],
$$

and insert it into Eq. (6), yielding

$$
\begin{aligned}
P_{A}= & \rho^{2} P_{m}+\tau^{2} P_{r}+\rho^{2} P_{m} r_{m}+\tau^{2} P_{r} r_{r} \\
& +2 \rho \tau \sqrt{\eta_{\text {het }} P_{m}\left(1+r_{m}\right) P_{r}\left(1+r_{r}\right)} \cos \left(\omega_{\text {het }} t-\varphi\right), \\
P_{B}= & \tau^{2} P_{m}+\rho^{2} P_{r}+\tau^{2} P_{m} r_{m}+\rho^{2} P_{r} r_{r} \\
& -2 \rho \tau \sqrt{\eta_{\text {het }} P_{m}\left(1+r_{m}\right) P_{r}\left(1+r_{r}\right)} \cos \left(\omega_{\text {het }} t-\varphi\right) .
\end{aligned}
$$

Factors of constant power are neglected in the following, since they do not add direct phase noise. Further, we simplify by using $r_{m, r} \ll 1$ so that $\sqrt{\left(1+r_{m}\right)\left(1+r_{r}\right)}=$ $\sqrt{1+r_{m}+r_{r}+r_{m} r_{r}} \approx \sqrt{1+r_{m}+r_{r}} \approx 1+\left(r_{m}+r_{r} / 2\right)$, yielding the simplified detection equations

$$
\begin{aligned}
P_{A} & =\underbrace{\rho^{2} P_{m} r_{m}+\tau^{2} P_{r} r_{r}}_{\text {If-RIN, port A }} \\
& \underbrace{+\left(r_{m}+r_{r}\right) \rho \tau \sqrt{\eta_{\text {het }} P_{m} P_{r}} \cos \left(\omega_{\text {het }} t-\varphi\right)}_{2 \mathrm{f}-\mathrm{RIN}, \text { port A }} \\
& \underbrace{+2 \rho \tau \sqrt{\eta_{\text {het }} P_{m} P_{r}} \cos \left(\omega_{\text {het }} t-\varphi\right)}_{\text {signal, port A }}, \\
P_{B} & =\underbrace{\tau^{2} P_{m} r_{m}+\rho^{2} P_{r} r_{r}}_{\text {If-RIN, port B }} \\
& \underbrace{-\left(r_{m}+r_{r}\right) \rho \tau \sqrt{\eta_{\text {het }} P_{m} P_{r}} \cos \left(\omega_{\text {het }} t-\varphi\right)}_{\text {2f-RIN, port B }} \\
& \underbrace{-2 \rho \tau \sqrt{\eta_{\text {het }} P_{m} P_{r}} \cos \left(\omega_{\text {het }} t-\varphi\right)}_{\text {signal, port B }} .
\end{aligned}
$$

At this point, we can already make important observations. Firstly, the term designated "1f-RIN" appears with the same sign in both output ports, and is approximately the same for well-matched 50:50 beam splitters. Therefore, this term can be suppressed with the balanced detection process defined above. Secondly, the term designated "2f-RIN" appears with the same phasing as the signal (and with half its amplitude) in both output ports and 
therefore cannot be reduced by balanced detection. Thirdly, the sum of equally labeled terms (sum of $1 \mathrm{f}-$ or 2f-RIN terms, respectively) that contain either $r_{m}$ or $r_{r}$ has to be performed linearly if the RIN is correlated between the two beams, and quadratically for uncorrelated RIN in the two beams. However, "1f-RIN" and "2f-RIN" terms are always considered uncorrelated with each other, since they occur at different frequencies and the noise is typically approximately white. Lastly, we see that all these noise contributions have been approximated as additive noise (phasors) to the main signal.

A very important aspect is that a phase meter is only sensitive in a narrow band around $f_{\text {het }}$, because the phase demodulation in Eq. (9) can be interpreted as a narrow filter around $f_{\text {het }}$. Therefore, only additive components in a bandwidth around the heterodyne frequency can cause phase noise.

a. The additive channel: If-RIN. When we look at the Fourier spectrum, we see that the terms labeled " $1 \mathrm{f}$ RIN" are only scaled by constant factors. Therefore these terms contribute RIN from near $1 f_{\text {het }}$, since higher- or lower-frequency components are filtered out through the demodulation process.

Note that the time delay $t_{k}$ included in the measurement beam also affects $1 \mathrm{f}$-RIN (at $f_{\text {het }}$ ), but since it is scaled by $f_{\text {het }}$ instead of $f_{m}$ or $f_{r}$, with $f_{\text {het }} \ll f_{m}$, we neglect it here and assume good correlation at the detector if RIN between the two beams was correlated at the start. However, for very large-scale interferometers like LISA, with significant light travel time differences, the postprocessing relies on correlated measurements, such that it may become relevant as a secondary noise effect.

$b$. The multiplicative channel: dc-RIN and 2f-RIN. The terms labeled "2f-RIN" behave differently since they carry the same sign as the signal. Moreover, they have an interesting frequency mixing property: they consist of the RIN itself $\left(r_{m}+r_{r}\right)$, which is then multiplied by half the heterodyne term. That heterodyne term has a frequency component at $f_{\text {het }}$. From Fourier analysis it follows for $r_{k}(t)$ with Fourier representation $\tilde{r}_{k}(f)$ that (see [11])

$$
\begin{aligned}
G_{k}(f) & :=\int r_{k} \cos \left(2 \pi f_{\text {het }} t\right) e^{-i 2 \pi f t} d t \\
& =\frac{1}{2}\left[\tilde{r}_{k}\left(f-f_{\text {het }}\right)+\tilde{r}_{k}\left(f+f_{\text {het }}\right)\right] .
\end{aligned}
$$

From Eq. (15) we see that the only possible coupling frequencies are small offset frequencies to dc, named $\epsilon$, and those around twice the heterodyne frequency, $f \approx\{0+$ $\left.\epsilon, 2 f_{\text {het }}\right\}$, because only such frequencies result in components near $f_{\text {het }}$ in the resulting spectrum (that is conjugate symmetric, since $r_{k}$ has only real values):

$$
G(0+\epsilon)=\frac{1}{2}\left[\tilde{r}_{k}\left(\epsilon-f_{\text {het }}\right)+\tilde{r}_{k}\left(\epsilon+f_{\text {het }}\right)\right],
$$

$$
G\left(2 f_{\text {het }}\right)=\frac{1}{2}\left[\tilde{r}_{k}\left(f_{\text {het }}\right)+\tilde{r}_{k}\left(3 f_{\text {het }}\right)\right]
$$

This up- and down-conversion is depicted in Fig. 2.

To investigate the dc terms further, we look at one specific RIN component at frequency $\epsilon$ with (long-term average) amplitude $r$ and a stochastic phase $\psi$, represented by $R=r \cos (2 \pi \epsilon t-\psi)$. We perform a similar operation as occurs in Eq. (14) by adding and multiplying a general signal $S=s \cos \left(2 \pi f_{\text {het }} t-\varphi\right)$ to demonstrate the effect:

$$
\begin{aligned}
S+\frac{R S}{2}= & S+\frac{r s}{4}\left[\cos \left[2 \pi\left(f_{\text {het }}-\epsilon\right) t-\varphi+\psi\right]\right. \\
& \left.+\cos \left[2 \pi\left(f_{\text {het }}+\epsilon\right) t-\varphi-\psi\right]\right] \\
= & \underbrace{\left(1+\frac{r}{2} \cos (2 \pi \epsilon t-\psi)\right)}_{\text {amplitude modulation }} s \cos \left(2 \pi f_{\text {het }} t-\varphi\right) .
\end{aligned}
$$
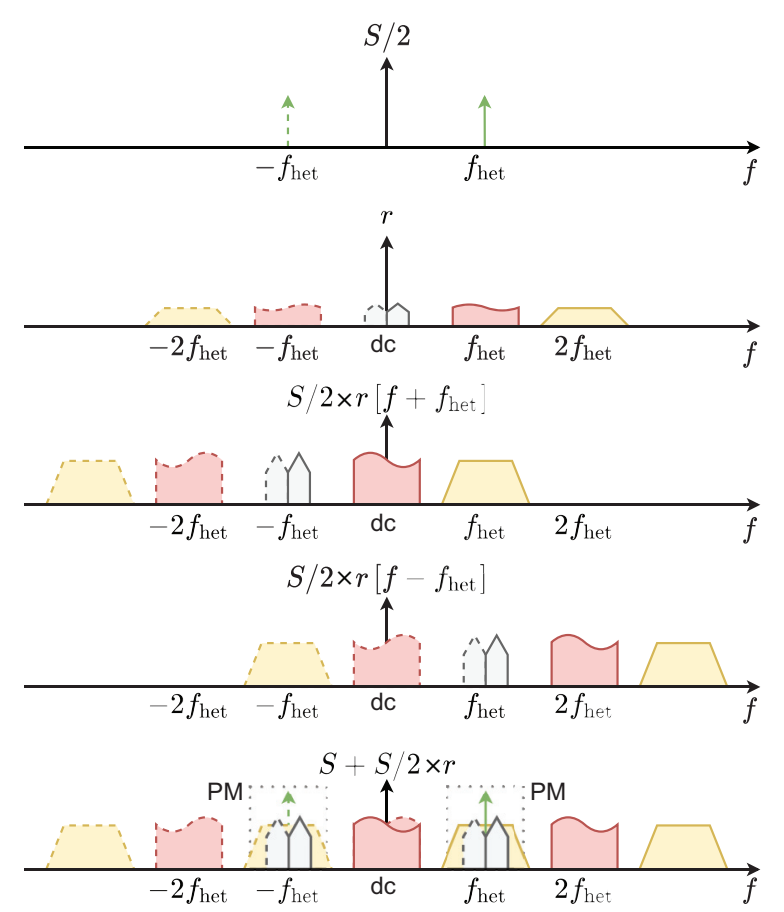

FIG. 2. Schematic illustrating the "2f-RIN" terms in Eq. (14) showing spectral mixing of (half) the signal beat term (first row) with RIN (second row). A real- valued signal has conjugate symmetry in its Fourier transform, which is indicated by the negative frequency range. As shown in Eq. (15), half of the noise power is shifted downwards (third row), while the other half is upconverted (fourth row). The total resulting spectrum (last row) is again conjugate symmetric. Therefore, RIN components from both $2 f_{\text {het }} \pm \epsilon$ and $0 \pm \epsilon$ (near dc) end up in the phase meter bandwidth. However, the dc components consist of conjugate phasors at $f_{\text {het }} \pm \epsilon$, meaning they only contribute amplitude noise and no direct phase noise. 
We conclude that RIN from near dc results in conjugate phasors around the heterodyne frequency. These phasors can only cause direct amplitude modulation of the signal and therefore do not contribute additive phase noise.

With that in mind, only the spectral contribution from $2 f_{\text {het }}$ remains relevant. With $G\left(2 f_{\text {het }}\right)$ it is clear that half of the RIN amplitude at $2 f_{\text {het }}$ is down-mixed to $f_{\text {het }}$ causing phase noise, while the other (nonrelevant) half is upconverted to $3 f_{\text {het }}$, which is not measured by the phase meter. The resulting scaling for $2 \mathrm{f}-\mathrm{RIN}$ (meaning $r_{m, r}$ near $2 f_{\text {het }}$ ) in port $\mathrm{A}$ and $\mathrm{B}$ is therefore

$$
2 \mathrm{f}-\mathrm{RIN}_{A, B}= \pm \frac{r_{m}+r_{r}}{2} \rho \tau \sqrt{\eta_{\text {het }} P_{m} P_{r}} .
$$

In conclusion, the only relevant terms causing RIN-tophase coupling at the photodetectors are

$$
\begin{aligned}
P_{A} & =\underbrace{\rho^{2} P_{m} r_{m}+\tau^{2} P_{r} r_{r}}_{\text {1f-RIN, port A }} \underbrace{+\frac{r_{m}+r_{r}}{2} \rho \tau \sqrt{\eta_{\text {het }} P_{m} P_{r}}}_{\text {2f-RIN, port A }} \\
& \underbrace{+2 \rho \tau \sqrt{\eta_{\text {het }} P_{m} P_{r}} \cos \left(2 \pi f_{\text {het }} t-\varphi\right)}_{\text {signal, port A }}, \\
P_{B} & =\underbrace{\tau^{2} P_{m} r_{m}+\rho^{2} P_{r} r_{r}}_{\text {1f-RIN, port B }} \underbrace{-\frac{r_{m}+r_{r}}{2} \rho \tau \sqrt{\eta_{\text {het }} P_{m} P_{r}}}_{\text {2f-RIN, port B }} \\
& \underbrace{-2 \rho \tau \sqrt{\eta_{\text {het }} P_{m} P_{r}} \cos \left(2 \pi f_{\text {het }} t-\varphi\right)}_{\text {signal, port B }} .
\end{aligned}
$$

It is important to note that for $1 \mathrm{f}-\mathrm{RIN}, r_{k}=r_{k}\left(f_{\text {het }}\right)$, and for 2f-RIN, $r_{k}=r_{k}\left(2 f_{\text {het }}\right)$.

We can already learn from these equations that the coupling coefficient for $2 \mathrm{f}-\mathrm{RIN}$ is roughly half the $1 \mathrm{f}-\mathrm{RIN}$ coefficient for equal beam powers, 50:50 beam splitters and high contrast $\left(\eta_{\text {het }} \approx 1\right)$.

In the following sections we derive the phase noise arising from $1 \mathrm{f}$ - and $2 \mathrm{f}$-RIN components, since the phase usually comprises the main observable of interest (as opposed to the induced noise current, for example).

We assume that the RIN spectrum is at least uncorrelated between frequencies as far apart as at least $\approx f_{\text {het }}$ and thus we can discuss $1 \mathrm{f}$ - and $2 \mathrm{f}$-RIN couplings separately. The total noise is then the quadratic sum of both $1 \mathrm{f}$ - and $2 \mathrm{f}-\mathrm{RIN}$ contributions.

We also develop formulas addressing the subtraction of a common mode reference phase, which allows us to find a working point that minimizes the coupling for both $1 \mathrm{f}$ - and 2f-RIN experimentally.

\section{A. RIN modeling approach}

In general, if the noise in the two beams is differential or uncorrelated RIN we write $r_{m}(t) \neq r_{r}(t)$. If only one laser source has been used it can only be caused by processes after the beams are split up (e.g., due to large differential time delays), or if two separate light sources have been used. Otherwise, if $r_{m}=r_{r}$, it is called common mode or correlated RIN. This defines whether both $r_{m, r}$ realizations are independent, adding them quadratically, or whether they are coherent, adding them linearly.

We begin to model the effect of RIN-to-phase coupling with a formal white noise Fourier series approach, because in a small band around the heterodyne frequency RIN is reasonably flat. While this can be used to derive the additional phase noise, we find it easier to simplify the calculations with phasor components. We formulate the full expression here and describe the second approach in the following section, which utilizes only two specific components of the Fourier series approach.

A narrow band white noise representation consists of $2 p$ sine waves around a center frequency $f_{c}$ with independent, instantaneous random amplitudes, $c_{k}$, and phases, $\psi_{k}$. This center frequency could be either $f_{c}=f_{\text {het }}$ or $f_{c}=2 f_{\text {het }}$. The amplitudes are given by $c_{i}=\left(2 \tilde{r}_{i}^{2} \Delta f\right)^{1 / 2}$ and are separated in frequency by $\Delta f \rightarrow 0 \mathrm{~Hz}$ as described in [16]. $\tilde{r}^{2}$ refers to the power spectral density (PSD) of the noise. Hence, this approach gives

$$
r_{m, r}\left(t, f_{c}\right)=\sum_{\substack{i=-p, i \neq 0}}^{p} c_{i} \cos \left[2 \pi\left(f_{c}+i \Delta f\right) t-\psi_{i}\right],
$$

with uniformly distributed random phase angles $\psi_{i}$ in $(0,2 \pi)$. The total noise power is $\left\langle r_{m, r}^{2}\right\rangle=2 p \Delta f \tilde{r}^{2}=2 B \tilde{r}^{2}$, because for white noise all $\tilde{r}_{i}$ converge to the same value for large enough sample sizes. The bandwidth $2 B$ represents in a typical phase meter readout the output sampling frequency of the phase measurement.

In the following section we show how one can simplify this approach.

\section{B. Phase noise from additive vector noise}

To simplify the calculations we utilize complex phasors to describe the general coupling mechanism of an additive vector (noise), as sketched in Fig. 3, and apply it to RINto-phase coupling afterwards. This is an adapted version of what can be found in [17]. A signal phasor, $S$, at frequency $\omega_{s}=2 \pi f_{s}$ and a small additive phasor, $V$, with frequency $\omega_{v}=2 \pi\left(f_{s}+\epsilon\right)$, is given by

$$
S=s e^{i\left(\omega_{s} t+\phi_{s}\right)}, \quad V=v e^{i\left(\omega_{v} t+\phi_{v}\right)},
$$

which results in a phasor

$$
R=S+V=r e^{i\left[\omega_{s} t+\phi_{r}(t)\right]},
$$

with

$$
r^{2}=s^{2}+v^{2}+2 s v \cos \left(2 \pi \epsilon t+\phi_{s}-\phi_{v}\right),
$$




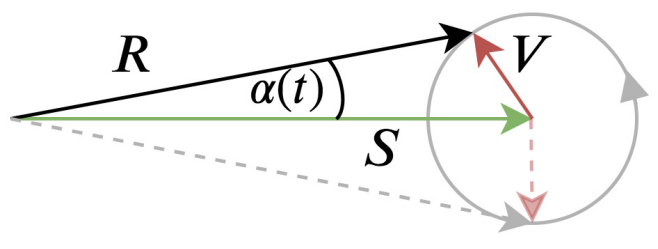

FIG. 3. Additive unwanted vectors, $V$, cause a phase deviation, $\alpha$, in the observable of interest, which is the phase of the signal, $S$.

$$
\phi_{r}=\arg \left(e^{i \phi_{s}}+\frac{v}{s} e^{i\left(2 \pi \epsilon t+\phi_{v}\right)}\right)
$$

We are interested in the phase variations of $\phi_{s}$, called $\alpha$, due to the presence of $V$ :

$$
\begin{aligned}
\phi_{r} & =\arg \left[e^{i \phi_{s}}\left(1+\frac{v}{S} e^{i\left(2 \pi \epsilon t+\phi_{v}-\phi_{s}\right)}\right)\right] \\
& =\phi_{S}+\underbrace{\arg \left(1+\frac{v}{S} e^{i\left(2 \pi \epsilon t+\phi_{v}-\phi_{S}\right)}\right)}_{=\alpha} .
\end{aligned}
$$

Therefore, the phase deviations, $\alpha$, are given by

$$
\begin{aligned}
& \alpha=\arg \left(1+\frac{v}{S} e^{i\left(2 \pi \epsilon t+\phi_{v}-\phi_{s}\right)}\right), \\
& =\arctan \left(\frac{\Im[1+\ldots]}{\operatorname{Re}[1+\ldots]}\right) \text {, } \\
& =\arctan \left(\frac{\frac{v}{s} \sin \left(2 \pi \epsilon t+\phi_{v}-\phi_{s}\right)}{1+\frac{v}{s} \cos \left(2 \pi \epsilon t+\phi_{v}-\phi_{s}\right)}\right) \text {, } \\
& \approx \frac{v}{S} \sin \left(2 \pi \epsilon t+\phi_{v}-\phi_{s}\right) \text {, }
\end{aligned}
$$

where we assume in the last step that the offending phasor, $V$, has a much smaller amplitude than $S$, that is, $v \ll s$. Figure 4 shows the resulting phase deviations. The variance or squared root mean square (rms) value is $\alpha_{\mathrm{rms}}^{2}=$ $\left(v^{2} / 2 s^{2}\right)$.

We can immediately extend this result for a phasor at a frequency $f_{s}-\epsilon$. Such a phasor would end up at $-\epsilon$ in the resulting phase spectrum, and be folded to $+\epsilon$ in a typical one-sided spectral calculation. This is important for the notion of band-limited noise around the signal, as it is present in real measurement systems. There, both sides of the signal contain a certain level of noise, uncorrelated with each other.

Using these considerations, we model the noise as follows. We assume band-limited white noise with a PSD value $n_{0}(f)$ and total noise power $N P=\int_{B} n_{0}(f) d f \approx$ $2 n_{0} B$, equally distributed to the left and right of the signal.

We already showed in the previous calculation that noise at a frequency $f_{s} \pm \epsilon$ ends up at frequency $\epsilon$ in the onesided phase spectrum. Therefore we can represent the noise for one particular frequency on the left and right side of the

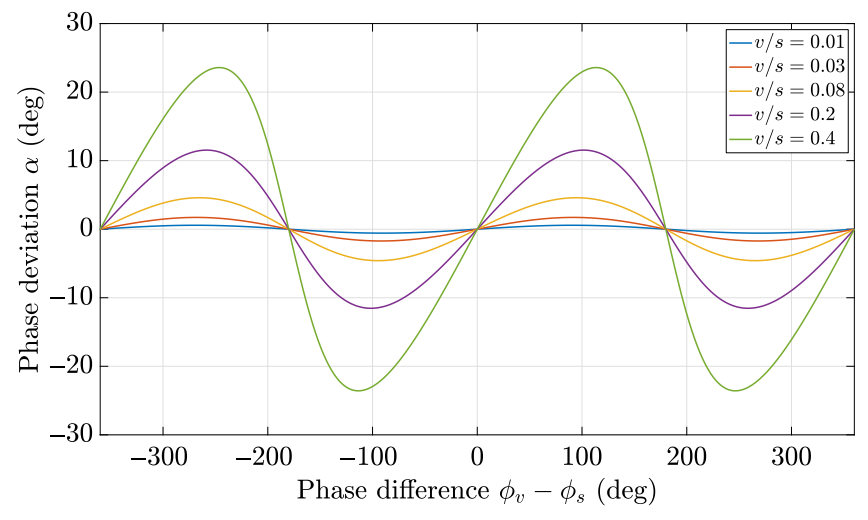

FIG. 4. Phase deviation in the signal phase for varying amplitude ratios of the signal and "noise" phasor with the nonapproximated phase formula.

signal by sine phasors $N_{1,2}$ with the white noise amplitude $n_{0}$ in a band of $b=1 \mathrm{~Hz}$. Both sine representations follow a uniform random phase distribution, since they represent uncorrelated noise:

$$
N_{1,2}=\sqrt{2 n_{0} b} \cos \left(\omega_{1,2} t+\psi_{1,2}\right),
$$

with $\omega_{1,2}=2 \pi\left(f_{s} \pm \epsilon\right)$ and equal noise power $N_{1,2, \mathrm{rms}}^{2}=$ $n_{0} b$. So, the total noise power measured in a PSD of the resulting phase at frequency $\epsilon$ would be $N_{1, \mathrm{rms}}^{2}+N_{2, \mathrm{rms}}^{2}=$ $2 n_{0} b=2 N_{1,2, \mathrm{rms}}^{2}$. Figure 5 shows a graphical representation of this.

We can simplify this even further and use only one single sine representation for both left and right phasors with additional scaling, since their noise power ends up at the
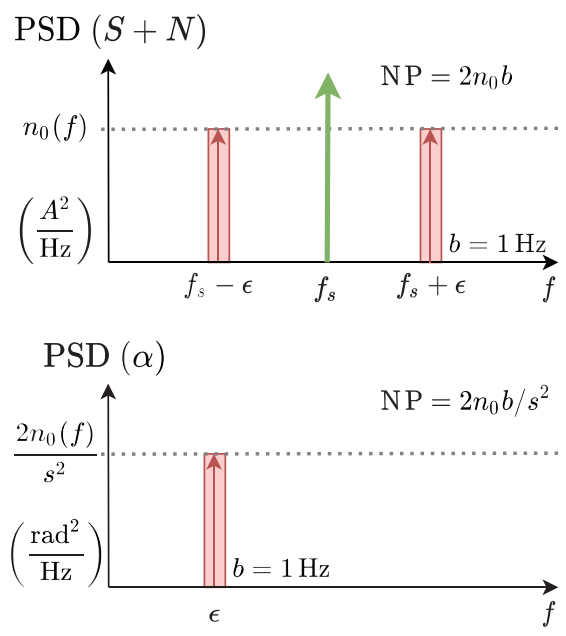

FIG. 5. Modeling band-limited additive white noise with sine waves in the PSD domain and the resulting spectral phase component. 
same frequency. This leads to a noise vector

$$
N=\sqrt{2} \sqrt{2 n_{0} b} \cos \left[2 \pi\left(f_{s}+\epsilon\right) t+\psi\right], N_{\mathrm{rms}}^{2}=2 n_{0} b .
$$

In the end, this is true for all frequencies in a bandwidth, $B$, that defines the sampling frequency of the output phase signal. Therefore, it is enough to propagate a general noise amplitude in this way to describe the whole resulting phase spectrum over the bandwidth $B$.

We can now describe the resulting phase noise from a general additive white noise process using Eq. (25d). A signal $S$ with amplitude $s$ and an additive small noise $N$ with amplitude $\sqrt{2} \sqrt{2 n_{0} b}$ at frequencies $f_{s} \pm \epsilon$ will lead to phase deviations of the form

$$
\alpha \approx \frac{\sqrt{2} \sqrt{2 n_{0} b}}{s} \sin \left(2 \pi \epsilon t+\psi-\phi_{s}\right)
$$

Calculating the variance, we find

$$
\alpha_{\mathrm{rms}}^{2}=\frac{2 n_{0} b}{s^{2}}
$$

which can be given in units of an amplitude spectral density (ASD) by dividing by $b=1 \mathrm{~Hz}$ and taking the square root:

$$
\tilde{\alpha}=\frac{\sqrt{2} \sqrt{n_{0}}}{s} .
$$

This result is true for any frequency, $\epsilon$. Therefore the frequency dependence is dropped and the result describes the whole output spectrum (for white noise).

This marks an important result for additive phase noise. Since $s / \sqrt{2}$ represents the signal rms value, for an approximately white noise source around a strong carrier signal we can calculate the resulting phase noise $\tilde{\alpha}$ with the formula

$$
\tilde{\alpha}=\frac{\text { Noise ASD }}{\text { Signal } \mathrm{rms}} \quad\left[\frac{\mathrm{rad}}{\sqrt{\mathrm{Hz}}}\right] .
$$

For the RIN terms in Eq. (19) the signal rms is given by $\rho \tau \sqrt{2 \eta_{\text {het, } A, B} P_{m} P_{r}}$. In the following, we calculate the phase noise, $\tilde{\alpha}$, for different $1 \mathrm{f}$ - and $2 \mathrm{f}-\mathrm{RIN}$ scenarios.

\section{1f-RIN phase coupling relations}

In this section we look at RIN at frequency $r_{k}=$ $r_{k}\left(1 f_{\text {het }}\right)$. For each output port, the $1 \mathrm{f}$-RIN has a differently scaled component due to the beam splitter parameters and power levels. Therefore, the formula for port B can be found by exchanging $\rho$ and $\tau$.

From Eqs. (19) and (31) it follows immediately that, for correlated RIN (i.e., $r_{m}=r_{r}=r$ ), the resulting phase noise in port $\mathrm{A}$ is given by

$$
\begin{aligned}
\tilde{\alpha}_{1 f, A} & =\frac{\rho^{2} P_{m} \tilde{r}_{m}+\tau^{2} P_{r} \tilde{r}_{r}}{\rho \tau \sqrt{2 \eta_{\text {het }} P_{m} P_{r}}} \\
& =\frac{\rho^{2} P_{m}+\tau^{2} P_{r}}{\rho \tau \sqrt{2 \eta_{\text {het }} P_{m} P_{r}}} \tilde{r}\left(1 f_{\text {het }}\right)=\frac{\sqrt{2} \times \tilde{r}\left(1 f_{\text {het }}\right)}{C_{A}}
\end{aligned}
$$

and for port $\mathrm{B}$

$$
\tilde{\alpha}_{1 f, B}=\frac{\tau^{2} P_{m}+\rho^{2} P_{r}}{\rho \tau \sqrt{2 \eta_{\text {het }} P_{m} P_{r}}} \tilde{r}\left(1 f_{\text {het }}\right)=\frac{\sqrt{2} \times \tilde{r}\left(1 f_{\text {het }}\right)}{C_{B}} .
$$

However, for uncorrelated RIN (i.e., $r_{m} \neq r_{r}$ ), the addition has to be performed quadratically, giving for port A

$$
\begin{aligned}
\tilde{\alpha}_{1 f, A} & =\frac{\sqrt{\left(\rho^{2} P_{m} \tilde{r}_{m}\right)^{2}+\left(\tau^{2} P_{r} \tilde{r}_{r}\right)^{2}}}{\rho \tau \sqrt{2 \eta_{\text {het }} P_{m} P_{r}}} \\
& \approx \frac{\sqrt{\left(\rho^{2} P_{m}\right)^{2}+\left(\tau^{2} P_{r}\right)^{2}}}{\rho \tau \sqrt{2 \eta_{\text {het }} P_{m} P_{r}}} \tilde{r}\left(1 f_{\text {het }}\right), \quad \text { if } \tilde{r}_{m} \approx \tilde{r}_{r},
\end{aligned}
$$

and for port B

$$
\begin{aligned}
\tilde{\alpha}_{1 f, B} & =\frac{\sqrt{\left(\tau^{2} P_{m} \tilde{r}_{m}\right)^{2}+\left(\rho^{2} P_{r} \tilde{r}_{r}\right)^{2}}}{\rho \tau \sqrt{2 \eta_{\mathrm{het}} P_{m} P_{r}}} \\
& \approx \frac{\sqrt{\left(\tau^{2} P_{m}\right)^{2}+\left(\rho^{2} P_{r}\right)^{2}}}{\rho \tau \sqrt{2 \eta_{\mathrm{het}} P_{m} P_{r}}} \tilde{r}\left(1 f_{\mathrm{het}}\right), \quad \text { if } \tilde{r}_{m} \approx \tilde{r}_{r} .
\end{aligned}
$$

Another interesting case appears if one of the beams has much higher intensity (e.g., $P_{r} \gg P_{m}$ ) while the RIN levels are somewhat comparable $\left(r_{m} \approx r_{r}\right)$. Then we get for correlated and uncorrelated RIN, respectively:

$$
\begin{array}{ll}
\tilde{\alpha}_{1 f, A}=\frac{\tau}{\rho} \sqrt{\frac{P_{r}}{2 \eta_{\text {het }} P_{m}}} \tilde{r}_{r}\left(1 f_{\text {het }}\right), & \text { if } P_{r} \gg P_{m}, \\
\tilde{\alpha}_{1 f, B}=\frac{\rho}{\tau} \sqrt{\frac{P_{r}}{2 \eta_{\text {het }} P_{m}}} \tilde{r}_{r}\left(1 f_{\text {het }}\right), & \text { if } P_{r} \gg P_{m} .
\end{array}
$$

In this case the phase noise is dominated by RIN from the more powerful beam.

\section{1f-RIN coupling relations after balanced detection}

Here, the simplified approach assumes identical processing and heterodyne efficiencies in the detection chains in port $\mathrm{A}$ and $\mathrm{B}$, and only allows a mismatch in the beam splitter parameters and beam powers. We gain an improvement in signal-to-noise ratio, since the signal is measured twice and added, while the noise is subtracted. As mentioned before, $2 \mathrm{f}-\mathrm{RIN}$ is not reduced and only the resulting $1 \mathrm{f}-\mathrm{RIN}$ is affected by balanced detection. 
The phase noise for correlated RIN after balanced detection is

$$
\tilde{\alpha}_{1 f, \Delta P}=\frac{\left|P_{m}\left(\rho^{2}-\tau^{2}\right)+P_{r}\left(\tau^{2}-\rho^{2}\right)\right|}{\rho \tau \sqrt{8 \eta_{\mathrm{het}} P_{m} P_{r}}} \tilde{r}\left(1 f_{\text {het }}\right),
$$

and for uncorrelated RIN

$$
\begin{aligned}
\tilde{\alpha}_{1 f, \Delta P}= & \frac{\sqrt{P_{m}^{2} \tilde{r}_{m}^{2}\left(\rho^{2}-\tau^{2}\right)^{2}+P_{r}^{2} \tilde{r}_{r}^{2}\left(\tau^{2}-\rho^{2}\right)^{2}}}{\rho \tau \sqrt{8 \eta_{\mathrm{het}} P_{m} P_{r}}} \\
\approx & \frac{\sqrt{P_{m}^{2}\left(\rho^{2}-\tau^{2}\right)^{2}+P_{r}^{2}\left(\tau^{2}-\rho^{2}\right)^{2}}}{\rho \tau \sqrt{8 \eta_{\mathrm{het}} P_{m} P_{r}}} \tilde{r}\left(1 f_{\mathrm{het}}\right) \\
& \text { if } \tilde{r}_{m} \approx \tilde{r}_{r} .
\end{aligned}
$$

We see that the resulting phase noise is minimized for $\rho^{2}=\tau^{2}$ (or $P_{m}=P_{r}$ when rearranging the terms). If that is the case, RIN is distributed equally in both ports and thus can be canceled to a large degree.

In the case where $P_{r} \gg P_{m}$ we find, for correlated and uncorrelated RIN, that

$$
\tilde{\alpha}_{1 f, \Delta P}=\left|\frac{\tau}{\rho}-\frac{\rho}{\tau}\right| \sqrt{\frac{P_{r}}{8 \eta_{\text {het }} P_{m}}} \tilde{r}_{r}\left(1 f_{\text {het }}\right) .
$$

\section{E. 1f-RIN common mode subtraction}

When subtracting another interferometric measurement that shares (some) of the same RIN, the subtraction can minimize the resulting phase noise. This is a technique for reducing common mode noises, typically implemented by a fixed beam "reference" interferometer. Note that we assume negligible time differences between interferometers.

Since the 1 f-RIN coupling depends on beam powers, heterodyne efficiencies and beam splitter coefficients, the general formula is not very compact. We derive a functional expression and simplify afterwards for common use-cases. In this section, we assume no differential time delay effects.

We denote the two interferometers involved by the subscript $n \in\{1,2\}$.

According to Eq. (27) we can represent $1 \mathrm{f}-\mathrm{RIN}$ by one component at $f_{\text {het }}+\epsilon$ as in

$$
r_{n, k}=2 \sqrt{r_{n, k}^{2} b} \cos \left[2 \pi\left(f_{\mathrm{het}}+\epsilon\right) t-\psi_{n, k}\right]
$$

where $r_{n, k}=r_{n, k}\left(1 f_{\text {het }}\right)$.

This term is scaled in the two interferometers by the following factors [see Eq. (19)]: $\rho_{n}^{2} P_{n, m}, \tau_{n}^{2} P_{n, m}, \rho_{n}^{2} P_{n, r}$, $\tau_{n}^{2} P_{n, r}$. Without loss of generality, we restrict ourselves to the case where only port A is used in either interferometer (otherwise $\rho$ and $\tau$ need to be exchanged appropriately):

$$
r_{n, m}=2 \rho_{n}^{2} P_{n, m} \sqrt{r_{n, m}^{2} b} \cos \left[2 \pi\left(f_{\text {het }, n}+\epsilon\right) t+\psi_{n, m}\right],
$$

$$
r_{n, r}=2 \tau_{n}^{2} P_{n, r} \sqrt{r_{n, r}^{2} b} \cos \left[2 \pi\left(f_{\mathrm{het}, n}+\epsilon\right) t+\psi_{n, r}\right] .
$$

The signal amplitude is

$$
s_{n}=2 \rho_{n} \tau_{n} \sqrt{\eta_{\text {het }, n} P_{n, m} P_{n, r}}
$$

and the signal phase is summarized by $-\varphi_{n}$. The resulting functional phase noise equations follow from Eq. (28); they are

$$
\begin{gathered}
\alpha_{n, m}=\frac{\rho_{n}}{\tau_{n}} P_{n, m} \sqrt{\frac{r_{n, m}^{2} b}{\eta_{\text {het }, n} P_{n, m} P_{n, r}}} \sin \left(2 \pi \epsilon t-\psi_{n, m}+\varphi_{n}\right), \\
\alpha_{n, r}=\frac{\tau_{n}}{\rho_{n}} P_{n, r} \sqrt{\frac{r_{n, r}^{2} b}{\eta_{\text {het }, n} P_{n, m} P_{n, r}}} \sin \left(2 \pi \epsilon t-\psi_{n, r}+\varphi_{n}\right) .
\end{gathered}
$$

To abbreviate, we define

$$
\begin{aligned}
g_{n, m} & :=\frac{\rho_{n}}{\tau_{n}} P_{n, m} \sqrt{\frac{r_{n, m}^{2} b}{\eta_{\text {het }, n} P_{n, m} P_{n, r}}}, \\
g_{n, r} & :=\frac{\tau_{n}}{\rho_{n}} P_{n, r} \sqrt{\frac{r_{n, r}^{2} b}{\eta_{\text {het }, n} P_{n, m} P_{n, r}}} .
\end{aligned}
$$

The general total phase noise after subtraction is

$$
\alpha_{\mathrm{tot}}=\alpha_{1, m}+\alpha_{1, r}-\left(\alpha_{2, m}+\alpha_{2, r}\right)
$$

whose terms have to be added quadratically for all uncorrelated noise terms, and linearly for all correlated terms.

To derive some practically useful equations, we introduce the following cases.In the first two, we assume that the mean power levels per beam are somewhat identical across the interferometers $\left(P_{1, m} \approx P_{2, m}=P_{m}, P_{1, r} \approx\right.$ $P_{2, r}=P_{r}$, but not necessarily $\left.P_{m}=P_{r}\right)$, that both recombination beam splitters are 50:50 $\left(\rho / \tau=\rho_{n} / \tau_{n} \approx \tau_{n} / \rho_{n}\right.$, and $\eta_{\text {het }, n} \approx \eta_{\text {het }}$ ), and differentiate then between (i) all beams sharing the same correlated RIN and (ii) pairwise uncorrelated $R I N$. In a third case (iii) we allow more variations between the interferometers, implying that the RIN cannot be fully subtracted. 
Case (i) is described by $r_{1, k}=r_{2, k}=r, \psi_{1, k}=\psi_{2, k}=\psi$, leading to

$$
\begin{aligned}
\alpha_{1, k} & -\alpha_{2, k} \\
& =g_{1, k}\left[\sin \left(2 \pi \epsilon t-\psi+\varphi_{1}\right)-\sin \left(2 \pi \epsilon t-\psi+\varphi_{2}\right)\right] \\
& =2 g_{1, k} \cos \left(2 \pi \epsilon t-\psi+\frac{\varphi_{1}+\varphi_{2}}{2}\right) \sin \left(\frac{\varphi_{1}-\varphi_{2}}{2}\right) .
\end{aligned}
$$

Setting $k=m$ and $k=r$ and adding both cases [in accordance with Eq. (47)] describes an ASD after taking the rms and dividing by $\sqrt{b}$ for each frequency $\epsilon$. Therefore it follows for white, correlated RIN that

$$
\tilde{\alpha}_{\mathrm{tot}}=\frac{\sqrt{2}\left(P_{m}+P_{r}\right)}{\sqrt{\eta_{\mathrm{het}} P_{m} P_{r}}} \tilde{r}\left(1 f_{\mathrm{het}}\right)\left|\sin \left(\frac{\varphi_{1}-\varphi_{2}}{2}\right)\right| .
$$

Case (ii) also features the same scaling factors across the interferometers, but uncorrelated RIN between the measurement and reference beams. This gives

$$
\begin{aligned}
\alpha_{\mathrm{tot}}= & g_{n, m}\left[\sin \left(2 \pi \epsilon t-\psi_{m}+\varphi_{1}\right)-\sin \left(2 \pi \epsilon t-\psi_{m}+\varphi_{2}\right)\right] \\
& +g_{n, r}\left[\sin \left(2 \pi \epsilon t-\psi_{r}+\varphi_{1}\right)-\sin \left(2 \pi \epsilon t-\psi_{r}+\varphi_{2}\right)\right] \\
= & 2\left[g_{n, m} \cos \left(2 \pi \epsilon t-\psi_{m}+\frac{\varphi_{1}+\varphi_{2}}{2}\right)\right. \\
& \left.+g_{n, r} \cos \left(2 \pi \epsilon t-\psi_{r}+\frac{\varphi_{1}+\varphi_{2}}{2}\right)\right] \\
& \times \sin \left(\frac{\varphi_{1}-\varphi_{2}}{2}\right) .
\end{aligned}
$$

In terms of an ASD it takes the form

$$
\tilde{\alpha}_{\text {tot }}=\sqrt{\frac{2\left(P_{m}^{2} \tilde{r}_{m}^{2}+P_{r}^{2} \tilde{r}_{r}^{2}\right)}{\eta_{\text {het }} P_{m} P_{r}}}\left|\sin \left(\frac{\varphi_{1}-\varphi_{2}}{2}\right)\right|,
$$

which simplifies further if $\tilde{r}_{m} \approx \tilde{r}_{r}=\tilde{r}$ to

$$
\tilde{\alpha}_{\text {tot }}=\sqrt{\frac{2\left(P_{m}^{2}+P_{r}^{2}\right)}{\eta_{\text {het }} P_{m} P_{r}}} \tilde{r}\left(1 f_{\text {het }}\right)\left|\sin \left(\frac{\varphi_{1}-\varphi_{2}}{2}\right)\right| .
$$

We see that even without balanced detection, the effect of $1 \mathrm{f}-\mathrm{RIN}$ can be nullified by subtracting a common RIN reference phase with the same interferometric operating point.

An experimentally relevant case is case (iii), where full RIN subtraction is not possible. Here we allow variations of the form $g_{2, m}=\left(1+\delta_{m}\right) g_{1, m}, g_{2, r}=\left(1+\delta_{r}\right) g_{1, r}$ between the interferometric ports. It is expected that the resulting maximum represents the sum of the individual coupling terms per interferometer, while the minimal coupling does not reduce to zero, but is rather limited by the difference of the induced phase noise per interferometer.
We use the following general approach to calculate the mean squared noise (after some algebra, similar to derivations above)

$$
\begin{aligned}
& \left\langle[a \sin (x+y)-a(1+\delta) \sin (x+z)]^{2}\right\rangle \\
& \quad=\left\langle a^{2}\right\rangle\left[\frac{\delta^{2}}{2}+(2+2 \delta) \sin \left(\frac{y-z}{2}\right)^{2}\right],
\end{aligned}
$$

where we have assumed that $x$ carries a random timedependence (as in $x=2 \pi \epsilon t-\psi$ ) and consider $y=$ $\varphi_{1}, z=\varphi_{2}$ as static for the time-scale of the averaging process. The RIN is hidden in $a$, which relates to the power noise per signal strength in the respective interferometer (i.e., the $g_{n, k}$ ).

If we assume in case (iii) uncorrelated RIN, we set either $a=g_{1, m}$ or $a=g_{2, m}$ and add the resulting mean squared terms from Eq. (3) (we drop the averaging symbols), giving

$$
\begin{aligned}
\alpha_{\mathrm{tot}}^{2}= & g_{1, m}^{2} \frac{\delta_{m}^{2}}{2}+g_{1, r}^{2} \frac{\delta_{r}^{2}}{2} \\
& +\left[g_{1, m}^{2}\left(2+2 \delta_{m}\right)+g_{1, r}^{2}\left(2+2 \delta_{r}\right)\right] \sin \left(\frac{\varphi_{1}-\varphi_{2}}{2}\right)^{2} .
\end{aligned}
$$

Now we can see that even for phase-matched interferometers $\left(\varphi_{1}=\varphi_{2}\right)$ there remains phase noise due to the amplitude mismatch as expected.

In terms of an ASD, for the remaining noise we get at the minimum (assuming division by $\sqrt{b}$ )

$$
\begin{aligned}
\tilde{\alpha}_{\mathrm{tot}, \min } & =\sqrt{g_{1, m}^{2} \frac{\delta_{m}^{2}}{2}+g_{1, r}^{2} \frac{\delta_{r}^{2}}{2}} \\
& =\frac{1}{\sqrt{2 \eta_{\text {het }, 1}}} \sqrt{\frac{\rho_{1}^{2}}{\tau_{1}^{2}} \frac{P_{1, m}}{P_{1, r}} \delta_{m}^{2} \tilde{r}_{m}^{2}+\frac{\tau_{1}^{2}}{\rho_{1}^{2}} \frac{P_{1, r}}{P_{1, m}} \delta_{r}^{2} \tilde{r}_{r}^{2} .}
\end{aligned}
$$

Looking at case (iii) and assuming correlated RIN, we have to consider cross terms between $r_{m}=r_{r}=r$. The mean squared expression is

$$
\begin{aligned}
\alpha_{\mathrm{tot}}^{2}= & g_{1, m}^{2} \frac{\delta_{m}^{2}}{2}+g_{1, r}^{2} \frac{\delta_{r}^{2}}{2}+g_{1, m} g_{1, r} \delta_{m} \delta_{r} \\
& +\left[4 g_{1, m} g_{1, r}+2 g_{1, m} g_{1, r}\left(\delta_{m}+\delta_{r}\right)\right. \\
& \left.+g_{1, m}^{2}\left(2+2 \delta_{m}\right)+g_{1, r}^{2}\left(2+2 \delta_{r}\right)\right] \sin \left(\frac{\varphi_{1}-\varphi_{2}}{2}\right)^{2}
\end{aligned}
$$


Unsurprisingly, the minimum here is larger due to the cross terms. The minimal noise ASD becomes

$$
\begin{aligned}
\tilde{\alpha}_{\text {tot, } \min } & =\frac{1}{\sqrt{2}}\left(g_{1, m} \delta_{m}+g_{1, r} \delta_{r}\right) \\
& =\frac{\frac{\rho_{1}}{\tau_{1}} P_{1, m} \delta_{m}+\frac{\tau_{1}}{\rho_{1}} P_{1, r} \delta_{r}}{\sqrt{2 \eta_{\text {het }, 1} P_{1, m} P_{1, r}}} \tilde{r}\left(1 f_{\text {het }}\right) .
\end{aligned}
$$

\section{F. 2f-RIN coupling relations}

This section deals with the down-converted RIN from twice the heterodyne frequency, $\tilde{r}_{k}=\tilde{r}_{k}\left(2 f_{\text {het }}\right)$. Using both Eqs. (19) and (31), we see that the resulting phase noise is independent of powers and contrasts. It only depends on the RIN level.

Correlated RIN with $r_{m}=r_{r}=r$ couples via

$$
\tilde{\alpha}_{2 f, A}=\frac{\tilde{r}_{m}+\tilde{r}_{r}}{\sqrt{8}}=\frac{1}{\sqrt{2}} \tilde{r}\left(2 f_{\text {het }}\right),
$$

while uncorrelated RIN $\left(r_{m} \neq r_{r}\right)$ follows

$$
\tilde{\alpha}_{2 f, A}=\frac{\sqrt{\tilde{r}_{m}^{2}+\tilde{r}_{r}^{2}}}{\sqrt{8}} \approx \frac{1}{2} \tilde{r}\left(2 f_{\mathrm{het}}\right), \quad \text { if } \tilde{r}_{m} \approx \tilde{r}_{r}=\tilde{r} .
$$

\section{G. 2f-RIN common mode subtraction}

In the $2 \mathrm{f}$-RIN common mode subtraction, the derivations are slightly more compact compared to the $1 \mathrm{f}-\mathrm{RIN}$ case, since the parameter space is reduced to correlation assumptions. Again, we use functional expressions with both amplitude and phase.

As for the 1f-RIN case, we assume two interferometers, labeled by the additional subscript $n \in\{1,2\}$. We look at one specific frequency component $\epsilon$ near $2 f_{\text {het }}$.

Following Eq. (27) we find the form

$$
r_{n, k}=2 \sqrt{r_{n, k}^{2} b} \cos \left[2 \pi\left(2 f_{\text {het }}+\epsilon\right) t-\psi_{n, k}\right] .
$$

According to Eq. (14) this is multiplied by $\rho \tau$ $\sqrt{\eta_{\text {het }, n} P_{n, m} P_{n, r}} \cos \left(2 \pi f_{\text {het }} t-\varphi_{n}\right)$. As mentioned before, only the component down-mixed to near $1 f_{\text {het }}$ couples through the phase meter, leaving

$$
\begin{aligned}
r_{n, k}= & \rho \tau \sqrt{\eta_{\text {het }, n} P_{n, m} P_{n, r}} \sqrt{r_{n, k}^{2} b} \\
& \times \cos \left[2 \pi\left(f_{\text {het }}+\epsilon\right) t-\psi_{n, k}+\varphi_{n}\right] .
\end{aligned}
$$

Therefore, using Eqs. (28) and (44), we find the functional form of the resulting phase noise

$$
\alpha_{n, k}=\frac{\sqrt{r_{n, k}^{2} b}}{2} \sin \left(2 \pi \epsilon t-\psi_{n, k}+2 \varphi_{n}\right) .
$$

Subtracting both interferometers yields

$$
\alpha_{\mathrm{tot}}=\alpha_{1, m}+\alpha_{1, r}-\left(\alpha_{2, m}+\alpha_{2, r}\right) .
$$

Now there are four possible RIN realizations. We restrict our analysis to two practical cases: (i) all beams have identical RIN; and (ii) pairwise identical RIN.

First, we assume that all four beams share the same correlated RIN with small enough light travel time differences, such that $r_{1, k}=r_{2, k}=r, \psi_{1, k}=\psi_{2, k}=\psi$, resulting in

$$
\begin{aligned}
\alpha_{\mathrm{tot}}= & \sqrt{r^{2} b}\left[\sin \left(2 \pi \epsilon t-\psi+2 \varphi_{1}\right)\right. \\
& \left.-\sin \left(2 \pi \epsilon t-\psi+2 \varphi_{2}\right)\right] \\
= & 2 \sqrt{r^{2} b} \cos \left(2 \pi \epsilon t-\psi+\varphi_{1}+\varphi_{2}\right) \sin \left(\varphi_{1}-\varphi_{2}\right) .
\end{aligned}
$$

By taking the rms and dividing by $\sqrt{b}$ we arrive at the corresponding ASD,

$$
\tilde{\alpha}_{\text {tot }}\left(2 f_{\text {het }}\right)=\sqrt{2} \times \tilde{r}\left(2 f_{\text {het }}\right)\left|\sin \left(\varphi_{1}-\varphi_{2}\right)\right|,
$$

which depends on the relative phase difference of the two interferometers, thus allowing the effect of $2 \mathrm{f}-\mathrm{RIN}$ to be nullified by adjusting the interferometric operating points.

For the second case, we simplify the formula by assuming that RIN is uncorrelated between the measurement and reference beams, but that the beams are shared in both interferometers. Again, we neglect travel time differences. This leads to $r_{1, m}=r_{2, m}=r_{m}, \psi_{1, m}=\psi_{2, m}=\psi_{m}$ and $\tilde{r}_{1, r}=\tilde{r}_{2, r}=\tilde{r}_{r}, \psi_{1, r}=\psi_{2, r}=\psi_{r}$.

Then

$$
\begin{aligned}
\alpha_{\mathrm{tot}}= & \frac{\sqrt{r_{m}^{2} b}}{2}\left[\sin \left(2 \pi \epsilon t-\psi_{m}+2 \varphi_{1}\right)\right. \\
& \left.-\sin \left(2 \pi \epsilon t-\psi_{m}+2 \varphi_{2}\right)\right] \\
& +\frac{\sqrt{r_{r}^{2} b}}{2}\left[\sin \left(2 \pi \epsilon t-\psi_{r}+2 \varphi_{1}\right)\right. \\
& \left.-\sin \left(2 \pi \epsilon t-\psi_{r}+2 \varphi_{2}\right)\right] \\
= & {\left[\sqrt{r_{m}^{2} b} \cos \left(2 \pi \epsilon t-\psi_{m}+\varphi_{1}+\varphi_{2}\right)\right.} \\
& \left.+\sqrt{r_{r}^{2} b} \cos \left(2 \pi \epsilon t-\psi_{r}+\varphi_{1}+\varphi_{2}\right)\right] \\
& \times \sin \left(\varphi_{1}-\varphi_{2}\right) .
\end{aligned}
$$

We find an ASD of

$$
\tilde{\alpha}_{\text {tot }}\left(2 f_{\text {het }}\right)=\frac{\sqrt{\tilde{r}_{m}^{2}+\tilde{r}_{r}^{2}}}{\sqrt{2}}\left|\sin \left(\varphi_{1}-\varphi_{2}\right)\right| .
$$

Assuming that $\tilde{r}_{m} \approx \tilde{r}_{r}=\tilde{r}$, we arrive at

$$
\tilde{\alpha}_{\text {tot }}\left(2 f_{\text {het }}\right)=\tilde{r}\left(2 f_{\text {het }}\right)\left|\sin \left(\varphi_{1}-\varphi_{2}\right)\right| \text {. }
$$




\section{H. Overview of simplified RIN coupling relations}

Using the previously derived equations, we summarize a few selected and simplified RIN-to-phase coupling relations in Table I with the following assumptions. The recombination beam splitter has an equal splitting ratio of $50 \%: 50 \%$. The band-limited white RIN can be correlated or uncorrelated between the beams, representing, for example, whether one (correlated) laser source or two (uncorrelated) laser sources have been used. When used, a common reference phase (e.g., from a reference interferometer) is assumed to have the same power levels, RIN contributions and contrast (quantities labeled $\varphi-\varphi_{R}$ ).

These relations show the coupling magnitude with individual contributions from $1 f_{\text {het }}$ and $2 f_{\text {het }}$. For uncorrelated RIN, approximately the same level $\tilde{r}_{m} \approx \tilde{r}_{r}$ is assumed.

It can be seen that the $2 f_{\text {het }}$ terms, which appear regardless of balanced detection, only depend on constant factors and are independent of the beam powers. For $1 f_{\text {het }}$ this is not the case and there is a dependence on the beam powers. It follows that with matched beam powers, $P_{m}=P_{r}$, and equal splitting at the recombination beam splitter, the $1 f_{\text {het }}$ contribution is a factor 2 higher than the $2 f_{\text {het }}$ contribution across all cases. However, the phase noise is magnified for imperfectly matched beam powers. This emphasizes the importance of balanced detection in precision metrology.

A rather typical scenario is the subtraction of a reference phase with common mode RIN. Here it can be seen that the error follows a sinusoidal pattern, depending on the relative phase relation. For $2 f_{\text {het }}$ the coupling strength becomes 0 if the two signals are in phase, and it becomes maximal for signals $90^{\circ}$ out of phase. For $1 f_{\text {het }}$ the coupling also disappears if the signals are in phase, but the maximal coupling is reached for signals $180^{\circ}$ out of phase. While 1f-RIN is well under control via balanced detection, this allows the minimizing of the $2 f_{\text {het }}$ contribution under certain circumstances, for example with perfectly aligned beams (i.e., a differential wavefront sensing offset of $0 \mathrm{rad}$ ) or vanishing testmass offsets in a LISA- or LPF-like measurement configuration.

\section{RIN SIMULATIONS}

Various simulations have been performed to verify the theoretical results stated in Table I. A simulator resembling the LPF style heterodyne phase meter readout system as described in [9] was developed and used to verify the theoretical derivations described above. The interfering beam powers, as they are disturbed by broadband white RIN of strength $\tilde{r}$, are fed with a sampling frequency of 50 $\mathrm{kHz}$ into SBDFTs with a discrete Fourier transform length of 500 samples at the heterodyne frequency of $1 \mathrm{kHz}$, generating a time-series phase output signal with a sampling frequency of $100 \mathrm{~Hz}$. Any light travel time delay on the RIN has been neglected in the simulation, since we assume $f_{m} \approx 300 \mathrm{THz} \gg f_{\text {het }}=1 \mathrm{kHz}$ and closely matched pathlengths in the interferometers. The resulting noise is measured, yielding an average phase noise ASD value. Different filters and noise realizations have been tested to verify the scenarios with both correlated and uncorrelated input noise for balanced and unbalanced detection. The common mode phase subtraction has been tested by simulating an additional reference interferometer signal that is subtracted from the main measurement. To simulate two different laser sources two independent noise realizations with the same average noise spectral density have been used.

Figure 6 shows the theoretical predictions (solid lines) and the numerical results (crosses) of the phase readout for the unbalanced, balanced and $1 f_{\text {het }}$ band-limited white noise cases after reference phase subtraction (see Table I, last row). If balanced detection is used, only the $2 f_{\text {het }}$ contribution causes phase disturbances, as can be seen by the green bottom trace. If only noise around $1 f_{\text {het }}$ is present, the coupling increases while the sinusoidal period doubles (blue trace in the middle). If only one output port is used or if balanced detection is inactive, both contributions have to be considered, which is shown by the light blue top curve, which corresponds to a quadratic addition of the $1 f_{\text {het }}$ and $2 f_{\text {het }}$ RIN terms. Both $1 f_{\text {het }}$ and $2 f_{\text {het }}$ terms together recover the unbalanced simulation. To separate the effect of $1 f_{\text {het }}$, a high-order bandpass filter has been used to suppress the $2 f_{\text {het }}$ component to a large degree. In all three cases two independent noise sources, averaged over 100 runs, have been used, representing two lasers with comparable properties. The average beam powers are kept identical at $1 \mathrm{~mW}$ with an input RIN ASD at an average level of $1 \times 10^{-6} / \sqrt{\mathrm{Hz}}$. A second interferometer is simulated as a reference interferometer, which allows the subtraction of its phase, leading to a sinusoidal noise dependence on the relative phase. The properties of the reference interferometer are identical to the measurement interferometer, except for a zero phase offset. RIN was correlated in the measurement and the reference interferometers, but uncorrelated between the measurement and reference beams.

Figure 7 shows the error magnification for all derived $1 f_{\text {het }}$ coupling coefficients (see Table I, column $1 f_{\text {het }}$ phase noise) as given by theory (solid lines) and simulation (crosses) for various input beam powers. The inputs are expressed with respect to the beam power ratio and the output is normalized by the minimal coupling strength of the smallest coupling ( $\varphi$ uncorr. RIN), which is reached when both beam powers are matched. The $2 f_{\text {het }}$ coupling is suppressed in this plot, since it does not depend on the power levels. It can be seen that, for unequally matched beam powers, the $1 f_{\text {het }}$ contribution may increase by orders of magnitude if imperfect or no balanced detection is applied. The symmetry and magnitude as given by the formulas are recovered. As expected, the maximal ratio between the correlated and uncorrelated cases is given by a factor of $\sqrt{2}$. The two cases with a reference phase subtraction have been 
TABLE I. Summary of the simplified RIN-to-phase coupling relations for a 50:50 recombination beam splitter and band-limited white RIN. The indices $m$ and $r$ refer to the measurement and reference beam, respectively. $P_{m, r}$ are the beam powers, while the RIN ASD values are described by $\tilde{r}\left(1,2 \times f_{\text {het }}\right)$. RIN can appear correlated (corr.) or uncorrelated (uncorr.) in the measured quantity, depending on the experimental configuration. For uncorrelated RIN its magnitude is assumed to have approximately the same value per beam, $\tilde{r}_{m} \approx \tilde{r}_{r}$. When a reference phase is subtracted, the beam power and RIN levels in the reference interferometer are assumed to be identical in $\varphi$ and $\varphi_{R}$. The total phase noise caused by RIN is given by the $2 f_{\text {het }}$ column alone if ideal balanced detection is used. However, in the case of unbalanced detection the $1 f_{\text {het }}$ and the $2 f_{\text {het }}$ terms have to be added quadratically.

\begin{tabular}{|c|c|c|}
\hline Measured quantity & $1 \mathrm{f}-\mathrm{RIN}$ phase noise $\left(\mathrm{rad} / \mathrm{Hz}^{1 / 2}\right)$ & $2 \mathrm{f}-\mathrm{RIN}$ phase noise $\left(\mathrm{rad} / \mathrm{Hz}^{1 / 2}\right)$ \\
\hline$\varphi\left(\right.$ corr. RIN $\left.r_{m}=r_{r}\right)$ & $\frac{P_{m}+P_{r}}{\sqrt{2 \eta_{\text {het }} P_{m} P_{r}}} \tilde{r}\left(1 f_{\text {het }}\right)$ & $\frac{1}{\sqrt{2}} \tilde{r}\left(2 f_{\text {het }}\right)$ \\
\hline$\varphi$ (uncorr. RIN: $r_{m} \approx r_{r}$ ) & $\sqrt{\frac{P_{m}^{2}+P_{r}^{2}}{2 \eta_{\text {het }} P_{m} P_{r}}} \tilde{r}\left(1 f_{\text {het }}\right)$ & $\frac{1}{2} \tilde{r}\left(2 f_{\text {het }}\right)$ \\
\hline$\varphi-\varphi_{R}\left(\right.$ corr. RIN: $\left.r_{m}=r_{r}\right)$ & $\frac{\sqrt{2}\left(P_{m}+P_{r}\right)}{\sqrt{\eta_{\text {het }} P_{m} P_{r}}} \tilde{r}\left(1 f_{\text {het }}\right)\left|\sin \left(\frac{\varphi-\varphi_{R}}{2}\right)\right|$ & $\sqrt{2} \tilde{r}\left(2 f_{\text {het }}\right)\left|\sin \left(\varphi-\varphi_{R}\right)\right|$ \\
\hline$\varphi-\varphi_{R}\left(\right.$ uncorr. RIN: $\left.r_{m} \approx r_{r}\right)$ & $\sqrt{\frac{2\left(P_{m}^{2}+P_{r}^{2}\right)}{\eta_{\text {het }} P_{m} P_{r}}} \tilde{r}\left(1 f_{\text {het }}\right)\left|\sin \left(\frac{\varphi-\varphi_{R}}{2}\right)\right|$ & $\tilde{r}\left(2 f_{\text {het }}\right)\left|\sin \left(\varphi-\varphi_{R}\right)\right|$ \\
\hline
\end{tabular}

calculated with a phase difference between measurement interferometer and reference interferometer of exactly $\pi$ to only record the maximal coupling coefficient. Every simulation is generated from new noise seeds, and for each point 50 averages have been used.

It should be noted that the phase demodulation processing affects the noise spectrum with its gain function. The exact transfer coefficient depends on the signal path and can be found by integrating the total noise power that is effectively propagating.

\section{LABORATORY INVESTIGATIONS}

In this section we present measurements of RIN-tophase coupling in a laboratory environment. First, we use a system that resembles the flight hardware of the LPF mission's optical metrology. In a second part, complementary measurements from a different optical testbed are shown.

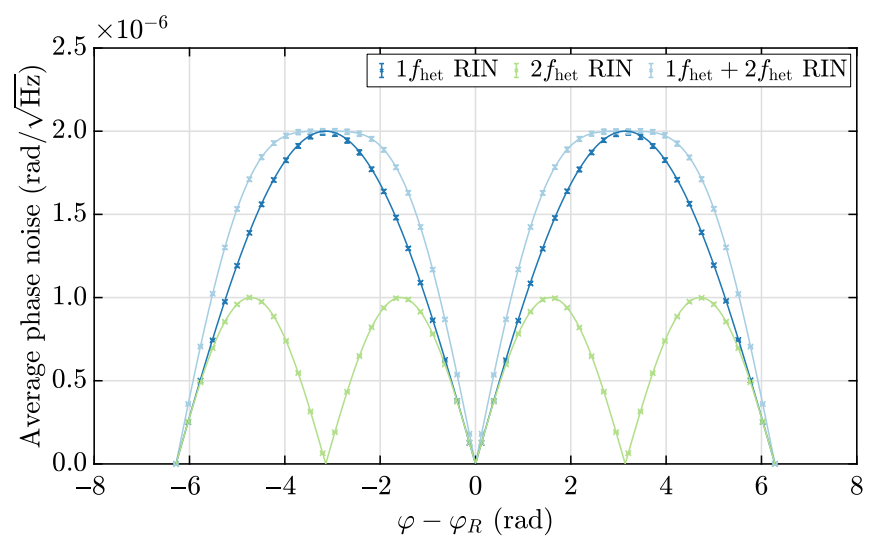

FIG. 6. Dependence of RIN-to-phase coupling on the differential phase between two interferometric measurements that share the same RIN. Simulation (crosses) and theory expectation (solid lines) are in agreement. A detailed description is given in the text.
The primary aim of the latter is to verify the LISA metrology chain, in particular its phase meter, but as well as to consider measurement of RIN-to-phase coupling.

\section{A. LPF testbed}

We give a brief overview of the system, explain the experiments and methods, and analyze the results.

\section{The laboratory}

This laboratory environment has previously been used as a demonstration testbed for the LPF mission. More detailed descriptions of the system, which is very similar to the actual flight hardware, can be found in $[7,9,18]$.

The light source is a single Nd:YAG NPRO laser at $1064 \mathrm{~nm}$ which is transmitted through a polarizationmaintaining fiber to the modulation stage. There, the light is split into two beams which are frequency-shifted by acousto-optic modulators (AOMs), such that their frequency difference is $f_{\text {het }}=1.6 \mathrm{kHz}$.

The ultra-stable LPF optical bench is placed in a vacuum chamber and contains four heterodyne interferometers. For our purposes, it is sufficient to use two of them. Similarly to Fig. 1, one interferometer propagates one beam to a (single) "test mass" (which consists of a piezo-controlled mirror), while another interferometer serves as a fixed reference with no movable parts.

We call the first interferometer $X_{1}$ with phase readout $x_{1}$, and the reference interferometer $X_{R}$ with phase readout $x_{R}$. The standard output (after balanced detection) is $o_{1}=$ $x_{1}-x_{R}$. However, the interferometer has two output ports $\mathrm{A}, \mathrm{B}$, such that it is also possible to look only at the A (or B) side, as in $o_{1, A}=x_{1, A}-x_{R, A}$.

Two active control loops keep the interferometers stable. An optical pathlength difference (OPD) loop uses piezo-controlled mirrors behind the AOMs to minimize pathlength fluctuations between the two beams that are 


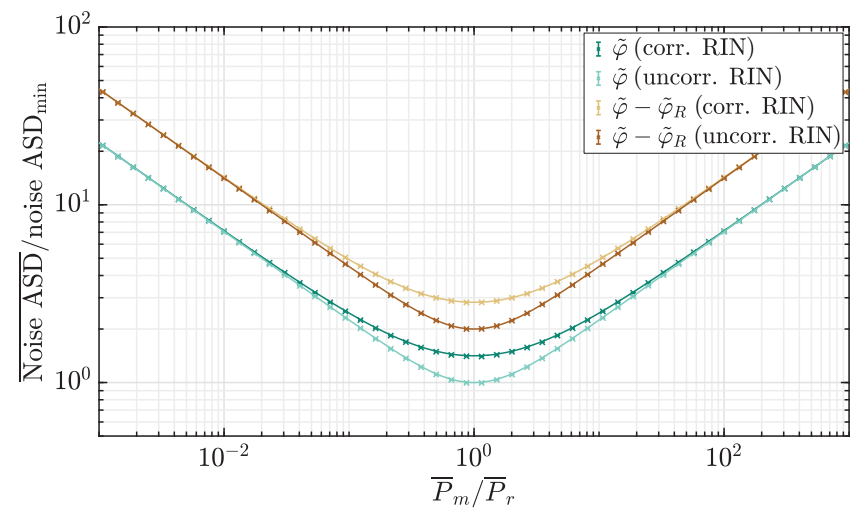

FIG. 7. Coefficients of $1 f_{\text {het }}$ RIN-to-phase coupling normalized to the minimal coupling. Simulation (crosses) and theory expectation (solid lines), evaluated at maximal coupling strength (sine factor equal to 1) in the phase subtraction case for equal RIN levels between the two beams. A detailed description is given in the text.

measured with the fixed reference interferometer. A second loop stabilizes the testmass mirror longitudinally via the readout of $o_{1, A}$.

Quadrant photodiodes (QPDs) are used to measure the optical signals, which in turn are digitized, summed to form a virtual single-element diode and filtered through various stages. A phase meter performs an SBDFT for each channel and provides the phase readout and mean powers. For single-beam RIN measurements we use another set of diodes and electronics.

For the experiment, we need to inject increased RIN in one of the beams, because the system is limited by other noises under nominal conditions. We use a modified advanced LIGO control and data system (CDS) [19] and subsequent digital-to-analog converter to modulate the laser amplitude via the AOMs. The CDS also operates the control loops and records data.

\section{Experimental design}

In the experiments, we look for the sinusoidal noise pattern in the phase spectrum as predicted by our previous calculations for both $1 \mathrm{f}$ - and $2 \mathrm{f}$-RIN (in separate experiments).

To create the necessary phase difference between the two interferometers, the testmass mirror is moved in discrete steps, which changes the phase of the measurement beam of the $X_{1}$ interferometer. The reference interferometer phase is kept locked at zero phase offset with the OPD loop.

Subtracting both measurements should reveal the sinusoidal noise structure. However, the amount of RIN in the system needs to be larger than the nominal readout noises, comprising quantization noise, signal conditioning

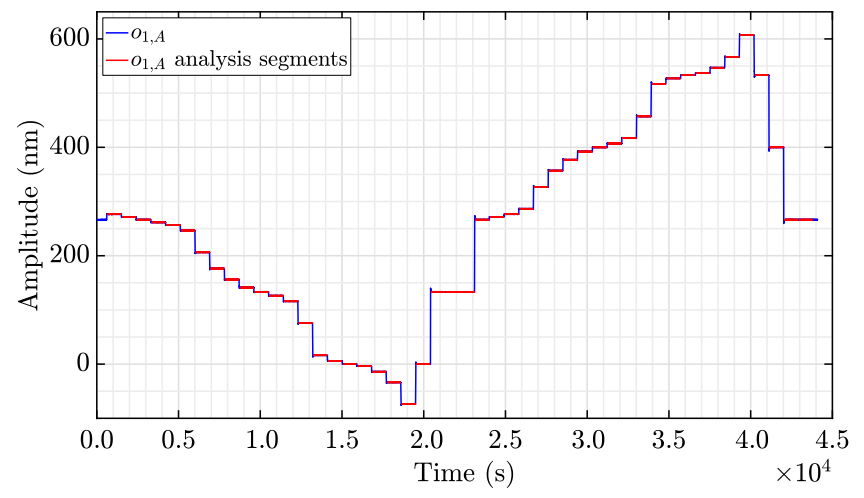

FIG. 8. Time series of the testmass steps during an injection of white RIN around $1 f_{\text {het }}$. Shown in blue is the subtracted signal of one output port, $o_{1, A}=x_{1, A}-x_{R, A}$. Marked in red are the stable periods selected for analysis. To create these steps, the testmass mirror was commanded to a new piezo set-point every $15 \mathrm{~min}$.

electronics noise, shot noise, frequency noise, mechanical vibrations, and other contributions.

To overcome this, we inject bandpassed white RIN into the reference beam in two distinct experiments. For the first experiment it is injected around $1 f_{\text {het }}$, while the second experiment targets $2 f_{\text {het }}$.

For the analysis of $1 \mathrm{f}-\mathrm{RIN}$, the beam powers after the beam splitter and contrasts need to be measured for each testmass set-point. These parameters enter the formulas predicting the resulting phase noise.

For $2 \mathrm{f}-\mathrm{RIN}$, only the beam powers (i.e., the injected RIN) have to be recorded, because the resulting noise shape is expected to be independent of other parameters.

Figure 8 shows the measured time series of the injected steps. The experiment consists of 47 steps with step sizes between 10 and $150 \mathrm{~nm}$. The typical hold duration at one step is $15 \mathrm{~min}$. These steps explore a phase space from approximately $-\pi / 4 \mathrm{rad}$ to $5 / 2 \pi \mathrm{rad}$.

\section{Signal chain and data processing}

The signal chain and data processing for the experiments is depicted in Fig. 9. RIN is injected via the "RIN injection chain," where white noise is generated digitally and added to the reference beam via its AOM driver in a band of $\approx 300 \mathrm{~Hz}$ around either $1 f_{\text {het }}$ or $2 f_{\text {het }}$, depending on the experiment.

Additionally, there are two distinct measurement chains, the "RIN measurement chain" and the "phase measurement chain." They are using different photodiodes, with separate analog-to-digital converter and subsequent data processing, as indicated.

With the "RIN measurement chain," RIN has to be measured at each testmass position, since it drifts over time. Therefore we record the single-beam powers on separate photodiodes with a sampling frequency of $64 \mathrm{kHz}$, which is later down-mixed and subsequently down-sampled. 


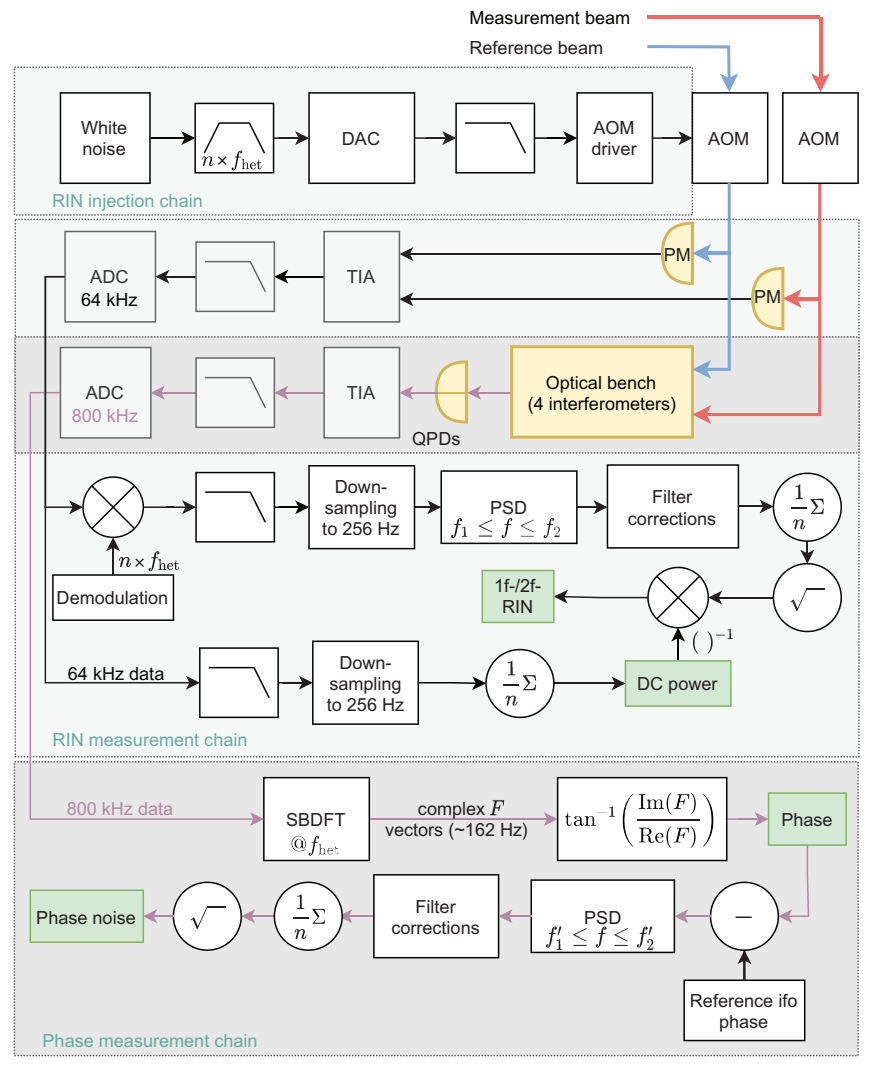

FIG. 9. Illustration of the signal and data flow for the RIN over testmass position measurements. White RIN is injected into the reference beam ("RIN injection chain"). For each testmass position it is propagated to power monitor photodiodes, digitized, demodulated, down-sampled and analyzed ("RIN measurement chain"). From both beams on the optical bench (for each testmass position), the heterodyne phase measurement is taken and extracted with an SBDFT via a separate signal path ("phase measurement chain," purple). Using both measurements, we are able to confirm the predicted coupling coefficients from injected RIN to measured phase noise for $1 \mathrm{f}-$ and $2 \mathrm{f}-\mathrm{RIN}$ coupling. Note that only the path for one QPD segment is shown here.

Both beams propagate through the optical bench and are brought to interference. Then the "phase measurement chain" measures the resulting heterodyne phase on the QPD of the $X_{1}$ and $X_{R}$ interferometers. For this, an SBDFT at the heterodyne frequency calculates sine and cosine components for each quadrant, summarized as complex $F$ vectors, that are implicitly down-sampled. In further data analysis, we calculate the phase noise with standard Fourier algorithms.

The anti-aliasing filters and transfer functions between the various stages have been characterized and are used to correct the data appropriately.

We compare the injected RIN in a flat frequency band (where RIN is the dominating noise source) with the corresponding frequency band (shifted by $f_{\text {het }}$ ) in the phase spectral density, using frequency bin averages.

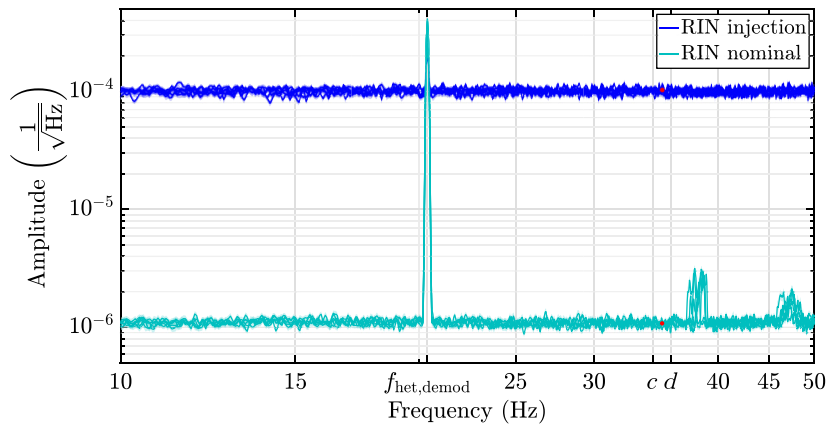

FIG. 10. RIN of the reference beam with and without additionally injected white noise for a few exemplary testmass set-points. The measurement has been taken according to the "RIN measurement chain" as depicted in Fig. 9. The calculated mean noise levels in the frequency band $[c, d]$ are indicated by the red points. The $2 \mathrm{f}-\mathrm{RIN}$ injections are not shown. The peak at $f_{\text {het,demod }} \approx n f_{\text {het }}-\left(n f_{\text {het }}-20 \mathrm{~Hz}\right)$ is likely originating from electrical cross-coupling between the AOM drivers. The $x$ axis is shifted due to the frequency used in the demodulation process.

In Fig. 10 we show the RIN of the reference beam with and without the white noise injection for multiple testmass positions, processed according to Fig. 9.

We chose a frequency band from $c=14 \mathrm{~Hz}$ to $d=$ $15.5 \mathrm{~Hz}$ that is offset to either $1 f_{\text {het }}$ or $2 f_{\text {het }}$ to determine each coupling coefficient.

For the spectral estimates we used 80 averages each from $50 \%$ overlapping $\mathrm{BH} 92$ windows. The direct RIN measurements are taken for the RIN-to-phase coupling predictions.

Figure 11 contains the resulting ASD of the measured phase noise for different testmass positions. We average the corresponding almost flat frequency band used for the RIN measurements (results marked in red).

Together with the testmass position (which is calculated as the mean phase value per segment, scaled to meters) we combine the results to typical phase noise over position plots, as presented in the next section.

\section{Experimental results}

Using the measured contributions, we calculate the phase noise predictions due to $1 \mathrm{f}$ - and $2 \mathrm{f}-\mathrm{RIN}$ for each experiment and testmass position with the equations from Secs. E and G. Since RIN is only injected in one beam, the expected phase noise equations take the form of uncorrelated RIN with expected residuals at the minimum due to imperfectly matched beam amplitudes in the two interferometers.

The RIN and phase noise are averaged as explained in the section above and plotted over the average testmass position for each of the 15 min segments.

The results for $1 \mathrm{f}-$ and $2 \mathrm{f}-\mathrm{RIN}$ injections, which depend on the testmass position (due to the subtraction of the correlated reference interferometer), are shown in Fig. 12. 


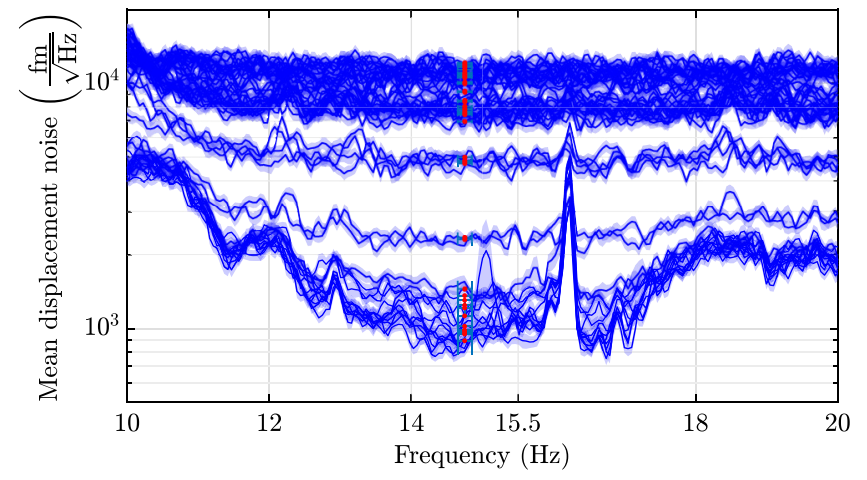

FIG. 11. Phase noise spectral densities at the different testmass positions during the $1 \mathrm{f}-\mathrm{RIN}$ injections. The figure shows the results from the "phase measurement chain" in Fig. 9 measured with the A side diodes. We see varying noise levels depending on the position. Marked in red are the pseudo-flat averages we chosen for analysis. They correspond to the range highlighted in Fig. 10 and avoid frequencies where other experimental influences are dominating. The spectrum during $2 \mathrm{f}-\mathrm{RIN}$ injections is not shown.

Here we only show the results for the frequency band $[c, d]$.

The prediction and the measured phase noise agree in shape and strength with the predications in most cases. However, some outliers remain in the data. Around the minima, there are also time-varying noise floor estimates visible, likely due to an unstable environment.

Furthermore, this shows, for experiments that rely on heterodyne-interferometry-based testmass readouts, the necessity to consider both $1 \mathrm{f}$ - and $2 \mathrm{f}-\mathrm{RIN}$ couplings.

\section{B. Measurements in hexagonal testbed}

Here, the complementary measurements in the hexagonal testbed are presented. This extends the validation of the presented model to the LISA frequency band using an independent setup.

\section{Experimental design}

As mentioned, the testbed was constructed to test the LISA metrology chain, in particular its phase meter, by implementing a three-signal test [20]. Its basic idea is to generate three cross-dependent interferometric phases, which cancel out in sum,

$$
\varphi_{\text {sum }}=\varphi_{1}+\varphi_{2}+\varphi_{3}=0
$$

This is achieved by interfering three lasers pairwise to obtain three beatnotes. The associated interferometric phases are then extracted by three separate phase meter channels. Hence, the residual noise floor after combing the extracted phases gives an estimate of the phase meter's performance. In the adoption to measure RIN-to-phase coupling, one of the seed laser beam's amplitudes is
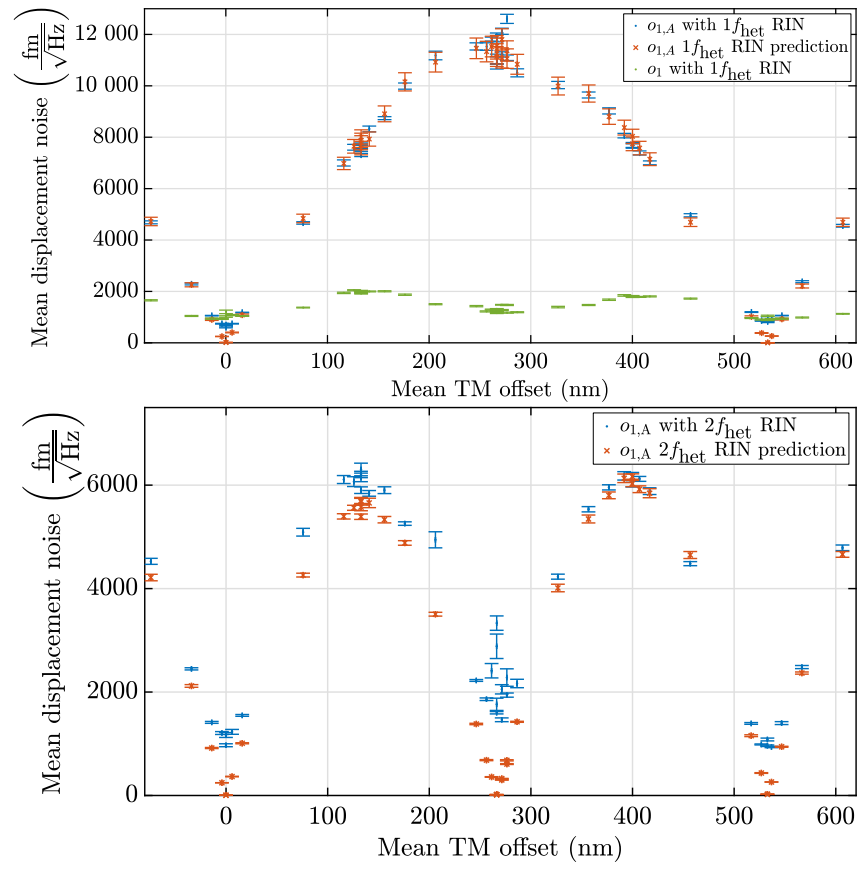

FIG. 12. Two plots showing the phase noise dependence of $1 \mathrm{f}$ - (top) and 2f-RIN-to-phase coupling (bottom). The phase on the $x$ axis is scaled to represent the mean testmass offset at a given step. We show the difference of the two A interferometer ports, $o_{1, A}=x_{1, A}-x_{R, A}$. From the direct RIN measurement at each step the predictions are calculated. The equations for uncorrelated RIN are used, since the injection affects only one beam. We find the expected sinusoidal behavior for the two cases in agreement with the theoretical predictions. In the top plot, the balanced measurement is also shown in green, where 1f-RIN subtracts while residual $2 \mathrm{f}-\mathrm{RIN}$ phase noise remains. The femtometer scale is used for the typical same-scale context of the LPF in-flight performance [7].

modulated using an electro-optical amplitude modulator (EOAM). The associated setup is shown in Fig. 13.

To investigate the impact of $1 \mathrm{f}-\mathrm{RIN}$, a white noise source is bandpassed around $2 \mathrm{MHz}$ and then added via the modulator as RIN. The numeric values of the three beatnote frequencies are chosen such that only one of them is within the frequency band around $2 \mathrm{MHz}$. The other two are kept well above $4 \mathrm{MHz}$. To investigate the impact of 2f-RIN, white noise around $4 \mathrm{MHz}$ can be added in the same fashion, while keeping the beatnote frequencies.

The plot in Fig. 14 shows the measurements of this setup, in particular in the LISA frequency band. A reference three-signal phase measurement without applied noise is shown in turquoise. It was taken shortly before the measurements with applied noise and is dominated towards lower frequencies by thermal fluctuations. Note that it yields a different performance compared to the measurement shown in [20], as it was taken shortly after the setup was changed and hence with increased thermal 


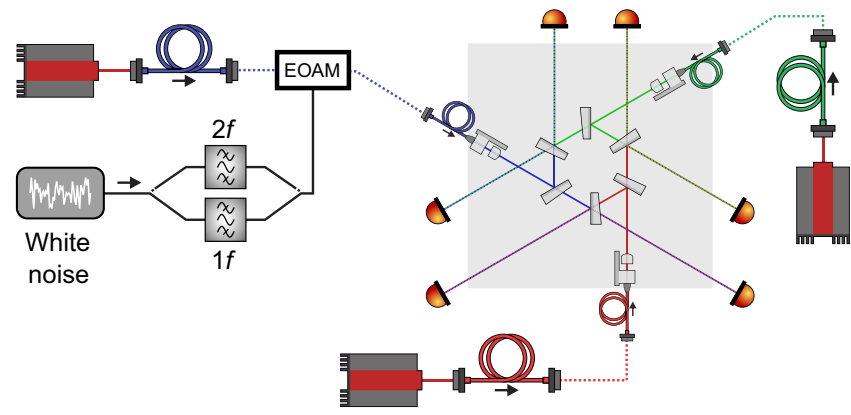

FIG. 13. Illustration of the optical setup to measure RINto-phase coupling utilizing a hexagonal optical bench. RIN is injected in one of the laser amplitudes. The beatnotes between the laser are controlled such that only one is affected by the noise. Then the combination of all three beatnotes is dominate by the RIN-to-phase coupling.

fluctuations. The blue dashed and the blue solid lines display the measurement with injected 1f-RIN and 2f-RIN, respectively. Their expected noise levels according to the presented model are drawn as red dashed and red solid lines, respectively. The comparison with the reference indicates that the blue curves are entirely dominated by the RIN-to-phase coupling. While the $1 \mathrm{f}-\mathrm{RIN}$ coupling fits the model nicely, the $2 \mathrm{f}-\mathrm{RIN}$ noise level is slightly below the expected values, likely due to an estimation error in the magnitude of the injected RIN. The green line shows the three-signal measurement of the same timeline as used for the blue dashed line, however with the final result being postprocessed with a balanced detection scheme. It shows the effectiveness of balanced detection for $1 \mathrm{f}$ RIN in the LISA band qualitatively. More details of these measurements can be found in [21].

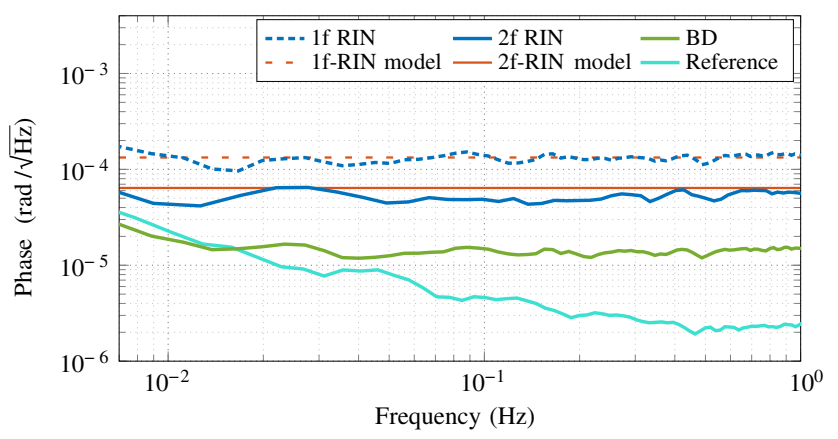

FIG. 14. Measurement showing RIN-to-phase coupling. With the turquoise line serving as reference measurement without coupling, the blue dashed and blue solid lines show the impact of the RIN-to-phase coupling for the $1 \mathrm{f}$ and $2 \mathrm{f}$ case, respectively. The expected values according to the model are presented in red dashed and red solid, respectively. The $1 \mathrm{f}$ case with successfully applied balanced detection is shown in green.

\section{SUMMARY}

We have derived, analyzed, simulated, and experimentally investigated $1 \mathrm{f}$ - and $2 \mathrm{f}$-RIN-to-phase couplings. Across all cases, good agreement between the theoretical predictions, the simulated and the experimental results is observed.

The laboratory results show that even in a realistic scenario with imperfect beam splitters, photodiodes and beam alignments the noise behavior can be well understood. We showed how RIN can impact the sensitivity of an interferometric testmass position readout.

RIN-to-phase coupling is a significant noise source for future space-based gravitational wave observatory missions such as LISA, where it is expected to cause approximately $10 \mathrm{pm} / \sqrt{\mathrm{Hz}}$ displacement noise if unmitigated. For high-precision heterodyne interferometers, stringent balancing requirements will have to guarantee good $1 f_{\text {het }}$ RIN suppression by design, and, the laser power stabilization around $2 f_{\text {het }}$ and its noise contribution need to be considered, since it cannot be subtracted by balanced detection.

\section{ACKNOWLEDGMENTS}

The authors would like to thank Brigitte Kaune, Sarah Paczkowski, Gudrun Wanner, Michael Born, MarieSophie Hartig, Olaf Hartwig, Ewan Fitzsimons, and JeanBaptiste Bayle for helpful discussions. We gratefully acknowledge support by the Deutsches Zentrum für Luftund Raumfahrt (DLR) with funding from the Bundesministerium für Wirtschaft und Technologie (project reference number 50 OQ 1801, based on work done under project reference numbers 50 OQ 1301 and 50 OQ 0601).

[1] Pau Amaro-Seoane et al., Laser Interferometer Space Antenna (2017). URL https://arxiv.org/abs/1702.00786.

[2] B. P. Abbott et al., GW150914: The Advanced LIGO Detectors in the Era of First Discoveries, Phys. Rev. Lett. 116, 131103 (2016).

[3] C. Affeldt et al., Advanced techniques in GEO 600, Classical Quantum Gravity 31, 224002 (2014).

[4] F. Acernese et al., The advanced virgo detector, J. Phys.: Conf. Ser. 610, 012014 (2015).

[5] Danielle M. R. Wuchenich et al., Laser link acquisition demonstration for the GRACE follow-on mission, Opt. Express 22, 11351 (2014).

[6] Gudrun Wanner, Gerhard Heinzel, Evgenia Kochkina, Christoph Mahrdt, Benjamin S Sheard, Sönke Schuster, and Karsten Danzmann, Methods for simulating the readout of lengths and angles in laser interferometers with Gaussian beams, Opt. Commun. 285, 4831 (2012).

[7] M. Armano et al., Sensor Noise in LISA Pathfinder: InFlight Performance of the Optical Test Mass Readout, Phys. Rev. Lett. 126, 131103 (2021). 
[8] M. Armano et al., Sub-Femto-G Free Fall for SpaceBased Gravitational Wave Observatories: LISA Pathfinder Results, Phys. Rev. Lett. 116, 231101 (2016).

[9] G. Heinzel et al., The LTP interferometer and phasemeter, Classical Quantum Gravity 21, S581 (2004).

[10] Klaus Abich et al., In-Orbit Performance of the GRACE Follow-On Laser Ranging Interferometer, Phys. Rev. Lett. 123, 031101 (2019).

[11] R. Stierlin, R. Bättig, P. D. Henchoz, and H. P. Weber, Excess-noise suppression in a fibre-optic balanced heterodyne detection system, Opt. Quantum Electron. 18, 445 (1986), ISSN 1572-817X.

[12] Gerald Hechenblaikner, Common mode noise rejection properties of amplitude and phase noise in a heterodyne interferometer, J. Opt. Soc. Am. A 30, 941 (2013).

[13] Gudrun Wanner and Gerhard Heinzel, Analytical description of interference between two misaligned and mismatched complete Gaussian beams, Appl. Opt. 53, 3043 (2014).

[14] Oliver Gerberding, PhD thesis, Leibniz University Hannover, 2014, URL https://www.repo.uni-hannover.de/ handle/123456789/8308.
[15] B. Shortt, L. Mondin, P. McNamara, K. Dahl, S. Lecomte, and Pedro Duque, LISA laser system and European development strategy. 11180.

[16] S. O. Rice, Mathematical analysis of random noise, Bell Syst. Tech. J. 23, 282 (1944), ISSN 0005-8580.

[17] W. P. Robins, Phase Noise in Signal Sources. Telecommunications. Institution of Engineering and Technology, (1984), URL https://digital-library.theiet.org/content/ books/te/pbte009e.

[18] H. Audley et al., The LISA pathfinder interferometry-hardware and system testing, Classical Quantum Gravity 28, 094003 (2011).

[19] Rolf Bork, AdvLigo CDS Design Overview (2010). URL https://dcc.ligo.org/LIGO-T0900612/public.

[20] Thomas S. Schwarze, Germán Fernández Barranco, Daniel Penkert, Marina Kaufer, Oliver Gerberding, and Gerhard Heinzel, Picometer-Stable Hexagonal Optical Bench to Verify LISA Phase Extraction Linearity and Precision, Phys. Rev. Lett. 122, 081104 (2019).

[21] Thomas S. Schwarze, PhD thesis, Leibniz University Hannover, 2018, URL https://www.repo.uni-hannover.de/ handle/123456789/4267. 